THE Astrophysical JouRnal, 476:717-729, 1997 February 20

(C) 1997. The American Astronomical Society. All rights reserved. Printed in U.S.A.

\title{
THE STRUCTURE OF THE GALACTIC MAGNETIC FIELD TOWARD THE HIGH-LATITUDE CLOUDS
}

\author{
Ana I. Gomez de Castro, ${ }^{1}$ Ralph E. Pudritz, ${ }^{2}$ and Pierre Bastien ${ }^{3}$ \\ Received 1995 December 22; accepted 1996 September 5
}

\begin{abstract}
We present the results of an optical polarization survey toward the galactic anticenter, in the area $5^{h} \geq \alpha \geq 2^{h}$ and $6^{\circ} \leq \delta \leq 12^{\circ}$. This region is characterized by the presence of a stream of high-velocity $\mathrm{H}$ I as well as high galactic latitude molecular clouds. We used our polarization data together with 100 $\mu \mathrm{m} I R A S$ maps of the region to study the relation between the dust distribution and the geometry of the magnetic field. We find that there is a correlation between the percent polarization and the $100 \mu \mathrm{m}$ flux such that $P(\%) \leq(0.16 \pm 0.05) F_{100}$. When the $I R A S$ flux is converted into $\mathrm{H}$ I column densities this becomes $P(\%) \leq(0.13 \pm 0.03) N_{20}-0.22$, which is consistent with previous interstellar medium studies on the relation between reddening and polarization. This correlation indicates that our survey is as deep as IRAS and that the magnetic field geometry does not change strongly with the optical depth in the lines of sight that we have studied. The implied lower limit to the distance of our survey is $500-700 \mathrm{pc}$ at galactic latitudes $b=-20^{\circ}$ and $100 \mathrm{pc}$ at $b=-50^{\circ}$.

Our main finding is that the magnetic field is perpendicular to the $\mathrm{H} \mathrm{I}$ high-velocity stream as well as the molecular cloud MBM 16 in the high-latitude region. The field is also perpendicular to the velocity gradient in the stream. Closer to the plane, the magnetic field is parallel to the dust filaments that extend like a plume toward the halo. Our observations indicate that the galactic magnetic field toward the highlatitude clouds is toroidal. We propose a model in which flux tubes that rise out of the galactic plane become force-free and predominantly toroidal at high latitudes. The high-latitude clouds may be gas streams that are falling back toward the galactic plane within such buoyant braided ropes of magnetic flux.
\end{abstract}

Subject headings: dust, extinction - infrared: ISM: continuum - ISM: magnetic fields ISM: molecules - polarization

\section{INTRODUCTION}

The completion of high-latitude $\mathrm{H} \mathrm{I}, I R A S$, and $\mathrm{CO}$ surveys has stimulated renewed interest in the question of the coupling between the disk of the Milky Way and its halo. The H I observations show that there are strong deviations from a uniform plane-parallel distribution of gas (see, e.g., reviews by Kulkarni \& Heiles 1988; Dickey \& Lockman 1990, hereafter DL). Large systems of arching filaments and shells are often observed. The $\mathrm{H}$ I and the IRAS $100 \mu \mathrm{m}$ fluxes are well correlated (De Vries \& Poole 1985; Boulanger \& Perault 1988; Martin et al. 1994). In fact, for extinctions of up to $2 \mathrm{mag}$, the $100 \mu \mathrm{m}$ brightness may be considered to be a good tracer of the $\mathrm{H}$ I column density to within a factor of 2 (Boulanger 1994). The highlatitude molecular clouds (Magnani, Blitz, \& Mundy 1985) appear to have physical conditions that are similar to the diffuse atomic gas. These transluscent clouds have masses of only a few hundred solar masses, and low densities (50-100 $\mathrm{cm}^{-3}$ ). They may simply be the regions of higher column density within the atomic gas that are sufficiently selfshielding from the UV radiation field $\left(N \geq 5 \times 10^{20} \mathrm{~cm}^{-2}\right)$ that molecules may form (see DL).

The dynamical state of high-latitude atomic gas has been intensively studied for decades. There is good evidence that

\footnotetext{
${ }^{1}$ Departamento de Astronomia y Geodesia, Facultad de CC. Matemáticas, Universidad Complutense de Madrid, Madrid, 28040, Spain.

${ }^{2}$ Department of Physics and Astronomy, McMaster University, Hamilton, ONT L8S 4M1, Canada.

${ }^{3}$ Observatoire du Mont Mégantic and Département de physique, Université de Montreal, B. P. 6128, Succ. Centre-ville, Montréal, Québec H3C 3J7, Canada.
}

the supershells are produced by multiple supernova explosions. However, many filamentary, high-latitude structures consist of discrete streams of gas. While the bulk of the $\mathrm{H} \mathrm{I}$ is at low LSR velocities $\left(\left|V_{\mathrm{LSR}}\right|<40 \mathrm{~km} \mathrm{~s}^{-1}\right)$, intermediate (up to $\left|V_{\mathrm{LSR}}\right|=90 \mathrm{~km} \mathrm{~s}^{-1}$ ) and high-velocity gas (up to $\left|V_{\mathrm{LSR}}\right|=500 \mathrm{~km} \mathrm{~s}^{-1}$ ) is found at high Galactic latitudes. These infalling clouds of low column densities were discovered in 1963 by Muller, Oort, \& Raimond because of their peculiar motions with respect to the galactic differential rotation. Since then the whole sky has been surveyed (Giovanelli 1980a; Bajaja et al. $1985\left[\delta \leq-10^{\circ}\right]$ and Hulsbosch \& Wakker $\left.1988\left[\delta \geq-18^{\circ}\right]\right)$. Approximately $18 \%$ of the sky is covered by high-velocity diffuse clouds detectable in $\mathrm{H}$ I. They are grouped in long chains and streams located preferentially in two directions of the sky. A comprehensive review on high-velocity clouds and their possible origins is given by Wakker \& Van Woerden (1991).

Several mechanisms, including infall from the outer halo (e.g., Mirabel 1980), have been proposed to explain the presence of gas at large distances from the galactic plane. Gas may be injected into the halo from the galactic disk through the agencies of supernova-driven fountain (Bregman 1980) or chimney flows (Norman \& Ikeuchi 1989). Similarly, the Parker instability (which is the magnetic analogue of the Rayleigh-Taylor instability wherein heavy fluid is supported by a light one) achieves similar effects. Buoyant arcades of magnetic flux tubes, originally layered in the galactic disk, push disk gas upward as the tubes rise. The gas then slides back down the field lines and gathers in magnetic valleys, (see, e.g., Blitz \& Shu 1980; Elmegreen 1982; Matsumoto et al. 1988; and Gómez de Castro \& Pudritz 1992). 
A neglected but potentially important clue about the nature of such disk/halo coupling can be gleaned from knowledge of the strength and structure of the galactic magnetic field at high latitudes. To take but one example, if the Parker instability is operative then one anticipates that streams of gas flow back down toward the galactic plane along magnetic field lines. If gas gathers in magnetic valleys, then the denser high-latitude molecular clouds could be regions where infalling streams converge and shock. Given that a microgauss field is sufficiently strong to affect the dynamics of the diffuse interstellar medium (ISM), it is clear that hydromagnetic processes must be taken into account.

Motivated by these ideas, we undertook an observing program to measure the optical polarization of selected stars in order to deduce the structure of the galactic magnetic field toward dynamically interesting regions, i.e., selected high-latitude gas streams. A preliminary report of our findings is found in Gómez de Castro, Pudritz, \& Bastien (1991). We selected the so-called anticenter stream or chain D (Wakker \& van Woerden 1991) because of its prominence, and because of the presence of both low- and highvelocity atomic gas as well as high-latitude molecular clouds (HLMCs) along its length. The anticenter stream is dominated by several elongated, filamentary $\mathrm{H}$ I clouds on whose borders one finds some well-studied, HLMCs such as MBM 16, 12, and 9. It is known that the HLMCs in our field lie within 65-95 pc (Hobbs et al. 1989). Thus we can be confident that many of the stars in the nearby fields are behind the clouds. At negative latitudes in the anticenter region there are two different dynamical components to the gas; the anticenter very high velocity (ACVHV) gas at LSR velocities of $\leq-150 \mathrm{~km} \mathrm{~s}^{-1}$, and anticenter high velocity gas at LSR velocities of $-90 \mathrm{~km} \mathrm{~s}^{-1}$ to $-150 \mathrm{~km} \mathrm{~s}^{-1}$ (Wakker \& van Woerden 1991 and references therein). The anticenter stream coincides in projection with the outer edge of the more significant components of the Gould Belt in the anticenter direction: the Taurus molecular clouds and the tip of the Belt in the Orion region. The detection of highly excited gas at this point suggests that at least part of the gas in the complex is being shocked (Mirabel 1982), so that the stream and these clouds may be physically associated as well.

In this paper we present the results of a linear polarization survey in the anticenter stream region $\left(5^{h} \geq \alpha \geq 2^{h}\right.$ and $6^{\circ} \leq \delta \leq 12^{\circ}$ ). We measured the optical polarization toward 136 stars in this region (§ 2). In order to separate contributions to the polarization from the galactic disk and that of the high-latitude structures, it is necessary to have a good fix on the column density of the gas at all latitudes. This was accomplished by using the IRAS $100 \mu \mathrm{m}$ map of the anticenter region since the interstellar clouds can be traced by their dust content through thermal emission. Using the polarization data and the IRAS $100 \mu \mathrm{m}$ map, we were able to determine the magnetic geometry toward the ACVHV and ACHV streams ( 3 ). The implications of our results for understanding the origins of the high-latitude clouds are discussed in $\S 4$.

\section{POLARIMETRIC OBSERVATIONS}

\subsection{The Program Stars}

Optical polarization data in the anticenter stream region are scant and consists of a few measured positions in the well-known Mathewson \& Ford (1970) survey. Similar surveys in this region, as compiled by Klare \& Neckel (1977), are also useful but do not focus on polarization toward the high-latitude clouds in particular. We selected several stellar fields along the ACHV stream on the basis of the H I column density from the Giovanelli (1980a) maps. Moreover, the red and blue POSS plates were searched for the more reddened regions, especially close to the galactic plane $\left(\alpha \sim 5^{h}\right)$ where the reddening was more apparent. The fields studied are indicated on the IRAS $100 \mu \mathrm{m}$ map shown in Figure 1 (Plates 8 and 9) (data retrieved from the Canadian Astronomy Data Centre). The $100 \mu \mathrm{m} I R A S$ flux extends like a plume from the galactic plane toward highgalactic latitudes. The substantial deviations in $N(\mathrm{H})$ from the mean galactic model (DL) are mirrored in the IRAS maps.

We divided our map into two regions. The prefix $\mathrm{B}$ is used for fields in the high-latitude stream ( $b$ from $-40^{\circ}$ to $-52^{\circ}$ ), and $\mathrm{A}$ for the fields close to the Orion region $\left(b \sim-20^{\circ}\right)$. We measured the polarization of $\sim 10$ stars per field up to a total of 136 targets. This survey samples stars as faint as visual magnitude 14.6 with polarization $P=0.8 \%$. All of our target stars are in the HST Guide Star Catalog (GSC). Their identification, coordinates, and magnitude taken from the HST GSC I, as well as their polarization measured in this work, are given in Table 1.

\subsection{Observations}

The observations were carried out in 1990 and 1991 at the Observatories of Mont Mégantic (Canada) and Calar Alto (Spain). The 1990 November data were obtained at the Mont Mégantic Observatory on a $1.6 \mathrm{~m}$ Ritchey-Chrétien telescope. The instrument was a two-channel photoelectric polarimeter similar to the one described by Angel \& Landstreet (1970). The modulator of this polarimeter is a Pockels cell.

The remaining observations were done at the Calar Alto Observatory. The first observing period was on the $1.2 \mathrm{~m}$ telescope at the Cassegrain focus in 1990 December. Two other observing periods followed during 1991 October and December with the $2.2 \mathrm{~m}$ telescope. The same polarimeter was used in all three runs; the modulator is a rotating achromatic half-wave plate (Proetel 1978).

All the observations were obtained with the same filter, a Schott RG-645. This filter, combined with the RCA 31034A photomultipliers in the two polarimeters, yields a passband $2450 \AA$-wide centered at $\lambda=7625 \AA$. Data from the two channels were averaged in order to get a single result. In both setups, the instruments automatically provide the percent polarization and position angle.

These values need to be corrected for the instrumental polarization. During the first night of each run the nonpolarized standards HD 21447, HD 14069, and HD 94851 were measured to calibrate the instrumental polarization. Further control measurements were done each night. The instrumental polarization was $0.1 \%$ and $0.2 \%$ in the 1990 and 1991 campaigns at Calar Alto, respectively. At Mégantic it was $0.06 \%$. For all runs, the instrumental polarization was subtracted from the data and the observational errors were adjusted accordingly. In addition, several polarized standards were observed in order to fix the origin of the position angles scale. The standards were: HD 14433, HD 23512, and HD 2619. Moreover, several program stars with low percent polarization were observed with the different instrumental set-ups to cross-check the calibrations. The 
TABLE 1

LOG OF DATA

\begin{tabular}{|c|c|c|c|c|c|c|c|c|}
\hline GSC ID & R. Asc. (1950) & Decl. & Magnitude & $P(\%)$ & $\sigma(P)$ & $\theta(\operatorname{deg})$ & $\sigma(\theta)$ & Field \\
\hline $00043.00033 \ldots \ldots$ & 20405.96 & +064747.5 & 11.85 & 0.17 & 0.07 & 0.8 & 11.4 & B1 \\
\hline $00043.00503 \ldots \ldots$ & 20422.44 & +065031.9 & 12.46 & 0.31 & 0.07 & 12.5 & 6.4 & B1 \\
\hline $00043.00329 \ldots \ldots$ & 20448.13 & +065907.3 & 12.50 & 0.25 & 0.07 & 22.2 & 7.5 & B1 \\
\hline $00043.00349 \ldots \ldots$ & 20449.02 & +065548.7 & 11.76 & 0.25 & 0.07 & 3.6 & 7.4 & B1 \\
\hline $00043.00005 \ldots \ldots$ & 20451.47 & +070225.0 & 11.40 & 0.20 & 0.06 & 20.6 & 9 & B1 \\
\hline $00043.00193 \ldots \ldots \ldots$ & 20538.16 & +070014.8 & 11.83 & 0.26 & 0.06 & 177.3 & 6.8 & B1 \\
\hline $00043.00413 \ldots \ldots$ & 20548.81 & +065124.9 & 12.19 & 0.28 & 0.06 & 2.9 & 6.5 & B1 \\
\hline $00043.00317 \ldots \ldots$ & 20607.01 & +065701.3 & 10.55 & 0.21 & 0.05 & 177.8 & 6.7 & B1 \\
\hline $00630.00806 \ldots \ldots$ & 20516.30 & +082334.2 & 10.12 & 0.12 & 0.04 & 126.8 & 9.0 & B2 \\
\hline $00630.00505 \ldots \ldots \ldots$ & 20535.51 & +082748.7 & 11.71 & 0.21 & 0.06 & 14.3 & 8.4 & $\mathrm{~B} 2$ \\
\hline $00630.00521 \ldots \ldots$ & 20544.42 & +082006.5 & 13.19 & 0.21 & 0.13 & 29.5 & 17.7 & B2 \\
\hline $00630.01047 \ldots \ldots \ldots$ & 20553.02 & +083357.9 & 11.88 & 0.27 & 0.07 & 178.5 & 7.5 & B2 \\
\hline $00630.00833 \ldots \ldots \ldots$ & 20553.54 & +081336.5 & 11.60 & 0.16 & 0.10 & 60.2 & 17.7 & B2 \\
\hline $00630.00992 \ldots \ldots \ldots$ & 20553.88 & +082459.8 & 13.04 & 0.12 & 0.07 & 18 & 15.7 & B2 \\
\hline $00630.00535 \ldots \ldots$ & 20605.69 & +082605.3 & 12.27 & 0.23 & 0.07 & 168.5 & 8.5 & B2 \\
\hline $00630.00781 \ldots \ldots$. & 20610.12 & +081852.5 & 10.87 & 0.08 & 0.06 & 140.5 & 24.5 & B2 \\
\hline $00630.01125 \ldots \ldots$ & 20611.69 & +081830.8 & 14.75 & 0.14 & 0.10 & 65.3 & 19.4 & B2 \\
\hline $00630.00657 \ldots \ldots \ldots$ & 20625.75 & +08 1339.2 & 12.37 & 0.16 & 0.10 & 163.0 & 18.3 & B2 \\
\hline $00635.00823 \ldots \ldots$ & 22154.42 & +101649.2 & 11.00 & 0.16 & 0.11 & 7.6 & 19.7 & B3 \\
\hline $00635.00958 \ldots \ldots$ & 22200.76 & +101340.0 & 12.92 & 0.20 & 0.12 & 35.7 & 16.4 & B3 \\
\hline $00635.01105 \ldots \ldots \ldots$ & 22205.31 & +100128.0 & 11.12 & 0.42 & 0.05 & 167.5 & 3.7 & B3 \\
\hline $00635.00655 \ldots \ldots$ & 22206.07 & +101252.1 & 12.33 & 0.31 & 0.18 & 179.2 & 16.7 & B3 \\
\hline $00635.00390 \ldots \ldots$ & 22206.92 & +101925.3 & 12.17 & 0.22 & 0.10 & 48.6 & 12.4 & B3 \\
\hline $00635.00782 \ldots \ldots \ldots$ & 22211.14 & +101818.8 & 13.81 & 1.12 & 0.35 & 21.5 & 8.9 & B3 \\
\hline $00635.00743 \ldots \ldots$ & 22226.24 & +102035.5 & 13.29 & 0.33 & 0.12 & 2.7 & 10.3 & B3 \\
\hline $00635.01140 \ldots \ldots$ & 22244.21 & +101424.1 & 12.75 & 0.61 & 0.21 & 38.2 & 10.1 & B3 \\
\hline $00635.00910 \ldots \ldots$. & 22248.98 & +100215.9 & 11.98 & 0.48 & 0.05 & 156 & 3.1 & B3 \\
\hline $00635.00730 \ldots \ldots$ & 22311.69 & +100908.6 & 10.33 & 0.50 & 0.05 & 176.1 & 3.1 & B3 \\
\hline $00632.00871 \ldots \ldots$ & 22549.01 & +083448.7 & 11.86 & 0.33 & 0.07 & 174.8 & 5.9 & B4 \\
\hline $00632.00714 \ldots \ldots \ldots$ & 22552.82 & +082729.2 & 12.68 & 0.26 & 0.09 & 149.5 & 10.6 & B4 \\
\hline $00632.00194 \ldots \ldots \ldots$ & 22558.98 & +083106.9 & 12.85 & 0.49 & 0.10 & 160.4 & 5.7 & B4 \\
\hline $00632.01078^{a} .$. & 22559.63 & +082626.0 & 8.870 & 0.06 & 0.038 & 161.4 & 20.0 & B4 \\
\hline $00632.00554 \ldots \ldots \ldots$ & 22603.11 & +083235.3 & 10.722 & 0.29 & 0.05 & 166.4 & 5.0 & B4 \\
\hline $00632.00166 \ldots \ldots$ & 22630.55 & +082433.2 & 12.68 & 0.18 & 0.10 & 42.5 & 15.6 & B4 \\
\hline $00632.00485 \ldots \ldots \ldots$ & 22632.13 & +083559.9 & 10.71 & 0.42 & 0.06 & 148.8 & 4.0 & B4 \\
\hline $00632.01103 \ldots \ldots \ldots$ & 22636.26 & +082745.0 & 14.78 & 2.51 & 0.10 & 170.1 & 1.1 & B4 \\
\hline $00632.00401 \ldots \ldots \ldots$ & 22637.90 & +083049.1 & 10.95 & 0.36 & 0.05 & 147.8 & 4.3 & B4 \\
\hline $00632.00797 \ldots \ldots \ldots$ & 22640.79 & +083614.5 & 10.98 & 0.22 & 0.06 & 7.6 & 8.6 & B4 \\
\hline $00639.01043 \ldots \ldots$. & 23322.53 & +072359.8 & 12.06 & 0.64 & 0.07 & 5.4 & 3.1 & B5 \\
\hline $00639.01233 \ldots \ldots \ldots$ & 23325.59 & +071701.9 & 11.63 & 0.41 & 0.07 & 178.5 & 5.0 & B5 \\
\hline $00639.01097 \ldots \ldots$. & 23336.20 & +073229.7 & 10.34 & 0.21 & 0.05 & 179.0 & 6.2 & B5 \\
\hline $00639.01138 \ldots \ldots \ldots$ & 23344.23 & +073354.6 & 11.37 & 0.18 & 0.05 & 7.3 & 7.2 & B5 \\
\hline $00639.00738 \ldots \ldots$. & 23436.00 & +073656.3 & 10.59 & 0.29 & 0.05 & 164.9 & 4.9 & B5 \\
\hline $00639.00605 \ldots \ldots \ldots$ & 23448.41 & +073159.4 & 12.17 & 0.52 & 0.07 & 15.0 & 3.7 & B5 \\
\hline $00639.00870 \ldots \ldots$ & 23451.63 & +073722.7 & 11.76 & 0.40 & 0.06 & 167.5 & 4.0 & B5 \\
\hline $00639.01167 \ldots \ldots \ldots$ & 23542.51 & +072033.8 & 12.57 & 0.35 & 0.07 & 172.4 & 5.9 & B5 \\
\hline $00640.00184 \ldots \ldots \ldots$ & 24349.59 & +0911 12.8 & 13.12 & 0.53 & 0.23 & 65.6 & 12.6 & B6 \\
\hline $00640.00237 \ldots \ldots$ & 24356.71 & +090834.6 & 12.17 & 0.41 & 0.07 & 12.4 & 5.2 & B6 \\
\hline $00640.00300 \ldots \ldots$ & 24407.33 & +090814.0 & 11.97 & 0.34 & 0.06 & 129.4 & 4.6 & B6 \\
\hline $00640.00805 \ldots \ldots \ldots$ & 24411.92 & +085801.1 & 10.652 & 0.15 & 0.05 & 158.6 & 8.5 & B6 \\
\hline $00640.00399 \ldots \ldots \ldots$ & 24415.56 & +090851.8 & 12.76 & 0.59 & 0.10 & 0.0 & 4.8 & B6 \\
\hline $00640.00270 \ldots \ldots$ & 24503.49 & +090558.2 & 12.86 & 0.84 & 0.07 & 6.2 & 2.4 & B6 \\
\hline $00644.00184 \ldots \ldots \ldots$ & 25247.24 & +102411.7 & 12.85 & 0.64 & 0.05 & 150.5 & 2.3 & B7 \\
\hline $00644.00372 \ldots \ldots$. & 25254.25 & +101821.9 & 11.86 & 0.43 & 0.06 & 130.3 & 4.0 & B7 \\
\hline $00644.00745 \ldots \ldots$ & 25259.65 & +101746.5 & 13.71 & 0.21 & 0.06 & 165.5 & 8.4 & B7 \\
\hline $00644.00514 \ldots \ldots$ & 25308.03 & +101144.4 & 14.62 & 0.81 & 0.15 & 167.4 & 5.2 & B7 \\
\hline $00644.00568 \ldots \ldots$. & 25312.51 & +100626.5 & 12.86 & 0.78 & 0.07 & 144.8 & 2.5 & B7 \\
\hline $00644.00534 \ldots \ldots \ldots$ & 25314.92 & +101534.6 & 11.72 & 0.98 & 0.06 & 130.0 & 1.6 & B7 \\
\hline $00644.00667 \ldots \ldots \ldots$ & 25324.22 & +101040.5 & 12.73 & 0.49 & 0.12 & 165.9 & 7 & B7 \\
\hline $00644.00364 \ldots \ldots \ldots$ & 25325.98 & +101854.0 & 12.66 & 0.68 & 0.10 & 154.9 & 4.3 & B7 \\
\hline $00644.00278 \ldots \ldots$ & 25328.71 & +101849.8 & 13.32 & 0.71 & 0.04 & 147.4 & 1.8 & B7 \\
\hline $00644.00132 \ldots \ldots \ldots$ & 25416.39 & +101652.3 & 10.73 & 0.10 & 0.05 & 163.9 & 16.4 & B7 \\
\hline $00644.00256 \ldots \ldots$ & 25418.31 & +100710.4 & 13.12 & 0.84 & 0.09 & 142.6 & 3.0 & B7 \\
\hline $00641.00104 \ldots \ldots \ldots$ & 25338.69 & +073128.3 & 12.95 & 0.38 & 0.07 & 133.9 & 5 & B8 \\
\hline $00641.00011 \ldots \ldots$. & 25342.44 & +072622.0 & 13.21 & 0.36 & 0.07 & 0.6 & 5.3 & B8 \\
\hline $00641.00285^{\mathrm{b}} \ldots \ldots$ & 25347.05 & +073445.2 & 9.897 & 0.28 & 0.07 & 19 & 6.9 & B8 \\
\hline $00641.00604 \ldots \ldots \ldots$ & 25354.44 & +073903.8 & 12.83 & 0.26 & 0.07 & 89.1 & 7.4 & B8 \\
\hline $00641.00474 \ldots \ldots \ldots$ & 25359.73 & +073254.4 & 10.66 & 0.27 & 0.06 & 166.5 & 6.4 & B8 \\
\hline $00641.00559 \ldots \ldots \ldots$ & 25400.67 & +073651.4 & 14.06 & 0.16 & 0.07 & 111.3 & 12.2 & B8 \\
\hline $00641.00810 \ldots \ldots$. & 25412.23 & +073723.9 & 12.37 & 0.19 & 0.07 & 116.8 & 11 & B8 \\
\hline $00641.00068 \ldots \ldots$. & 25413.66 & +072710.3 & 10.70 & 0.38 & 0.07 & 112.8 & 4.9 & B8 \\
\hline $00641.00671 \ldots \ldots$. & 25450.65 & +072949.4 & 13.37 & 0.60 & 0.07 & 49.5 & 3.4 & B8 \\
\hline $00061.00367 \ldots \ldots$. & 30603.82 & +070436.9 & 12.25 & 1.31 & 0.07 & 9 & 1.5 & B9 \\
\hline
\end{tabular}


TABLE 1-Continued

\begin{tabular}{|c|c|c|c|c|c|c|c|c|}
\hline GSC ID & R. Asc. (1950) & Decl. & Magnitude & $P(\%)$ & $\sigma(P)$ & $\theta(\operatorname{deg})$ & $\sigma(\theta)$ & Field \\
\hline $00061.00221 \ldots \ldots$. & 30617.23 & +071754.6 & 11.48 & 0.77 & 0.07 & 11.1 & 2.5 & B9 \\
\hline $00061.00177 \ldots \ldots$. & 30622.44 & +0704 46.7 & 11.80 & 1.13 & 0.05 & 6.4 & 1.3 & B9 \\
\hline $00061.00039 \ldots \ldots$ & 30652.72 & +070601.3 & 11.67 & 0.29 & 0.06 & 26 & 6.4 & B9 \\
\hline $00061.00115 \ldots \ldots$ & 30710.16 & +070821.6 & 11.85 & 1.71 & 0.07 & 10.8 & 1.2 & B9 \\
\hline $00062.00113 \ldots \ldots$. & 30743.72 & +071550.8 & 11.92 & 0.22 & 0.07 & 179.2 & 8.8 & B9 \\
\hline $00648.01070 \ldots \ldots$. & 30626.42 & +093328.9 & 10.96 & 0.96 & 0.05 & 24.9 & 1.6 & B10 \\
\hline $00648.01255 \ldots \ldots \ldots$ & 30640.04 & +09 3521.4 & 11.57 & 0.58 & 0.11 & 6.4 & 5.4 & B10 \\
\hline $00648.01236 \ldots \ldots$ & 30640.77 & +093556.1 & 11.15 & 0.68 & 0.07 & 11.6 & 3.1 & B10 \\
\hline $00651.01168 \ldots \ldots \ldots$ & 30710.98 & +095037.3 & 11.96 & 0.39 & 0.06 & 60.8 & 4.4 & B10 \\
\hline $00648.01052 \ldots \ldots$. & 30721.54 & +094356.0 & 12.49 & 0.16 & 0.07 & 149.5 & 13.1 & B10 \\
\hline $00648.01234 \ldots \ldots \ldots$ & 30724.52 & +09 3739.4 & 10.50 & 0.34 & 0.06 & 6.1 & 4.6 & B10 \\
\hline $00648.01175 \ldots \ldots$ & 30752.88 & +094426.5 & 10.19 & 0.40 & 0.04 & 143.9 & 2.7 & B10 \\
\hline $00648.01139 \ldots \ldots$. & 30829.01 & +094717.9 & 13.43 & 0.60 & 0.08 & 18.8 & 3.6 & B10 \\
\hline $00648.01265 \ldots \ldots \ldots$ & 30841.86 & +09 3057.7 & 15.27 & 0.75 & 0.17 & 12.3 & 6.4 & B10 \\
\hline $00651.00944 \ldots \ldots$. & 30850.08 & +095308.4 & 12.47 & 0.93 & 0.07 & 31.4 & 2 & B10 \\
\hline $00687.01105 \ldots \ldots$. & 44435.02 & +100145.5 & 12.87 & 2.31 & 0.12 & 26 & 1.5 & A1 \\
\hline $00687.01278 \ldots \ldots \ldots$ & 44448.63 & +095942.8 & 11.86 & 0.48 & 0.06 & 20.9 & 3.3 & A1 \\
\hline $00687.01010 \ldots \ldots$ & 44518.61 & +095732.8 & 12.40 & 2.18 & 0.07 & 69.8 & 1 & A1 \\
\hline $00687.01211 \ldots \ldots$ & 44519.33 & +095926.7 & 10.00 & 2.11 & 0.05 & 68.3 & 0.7 & A1 \\
\hline $00687.00498 \ldots \ldots \ldots$ & 44525.58 & +105539.9 & 11.91 & 1.94 & 0.13 & 70.3 & 1.9 & A1 \\
\hline $00687.00921 \ldots \ldots$. & 44535.19 & +101446.0 & 12.70 & 2.75 & 0.06 & 62.4 & 0.6 & A1 \\
\hline $00687.00411 \ldots \ldots \ldots$ & 44601.53 & +100330.0 & 11.75 & 2.01 & 0.07 & 66.8 & 0.9 & $\mathrm{~A} 1$ \\
\hline $00687.00762 \ldots \ldots$. & 44602.58 & +095752.0 & 11.98 & 0.31 & 0.05 & 17.3 & 5 & A1 \\
\hline $00687.01461^{\mathrm{c}} \ldots \ldots$ & 44608.29 & +101753.5 & 9.93 & 2.85 & 0.02 & 56.8 & 0.2 & A1 \\
\hline $00687.01466 \ldots \ldots$ & 44618.66 & +100208.5 & 11.77 & 2.17 & 0.04 & 66.4 & 0.5 & A1 \\
\hline $00687.01135 \ldots \ldots$. & 44619.39 & +100832.6 & 11.99 & 1.82 & 0.08 & 65.8 & 1.2 & A1 \\
\hline $00687.00654 \ldots \ldots$. & 44618.20 & +10 1729.1 & 11.42 & 2.69 & 0.05 & 59.9 & 0.6 & A1 \\
\hline $00687.00912 \ldots \ldots$. & 44635.11 & +102247.1 & 10.46 & 1.78 & 0.06 & 61.9 & 1 & A1 \\
\hline $00687.00912 \ldots \ldots \ldots$ & 44635.11 & +102247.1 & 10.46 & 2.65 & 0.11 & 59.5 & 1.2 & A1 \\
\hline $00683.01133 \ldots \ldots$ & 44700.56 & +074754.1 & 12.01 & 0.77 & 0.07 & 38.7 & 2.5 & A2 \\
\hline $00683.00565 \ldots \ldots$. & 44700.69 & +072611.4 & 11.40 & 0.61 & 0.06 & 37.9 & 2.9 & $\mathrm{~A} 2$ \\
\hline $00683.00637 \ldots \ldots$ & 44701.04 & +074042.1 & 12.42 & 0.57 & 0.06 & 37.5 & 2.9 & $\mathrm{~A} 2$ \\
\hline $00683.00663 \ldots \ldots \ldots$ & 44707.58 & +073803.0 & 11.44 & 0.72 & 0.06 & 36.7 & 2.5 & $\mathrm{~A} 2$ \\
\hline $00683.01083 \ldots \ldots$. & 44741.05 & +074853.8 & 12.45 & 0.80 & 0.07 & 37.5 & 2.7 & A2 \\
\hline $00683.00701 \ldots \ldots$ & 44750.75 & +073408.0 & 11.34 & 0.68 & 0.05 & 38.9 & 2 & $\mathrm{~A} 2$ \\
\hline $00684.00870 \ldots$ & 44833.44 & +074923.5 & 11.32 & 0.34 & 0.07 & 38.6 & 5.7 & A2 \\
\hline $00684.01019 \ldots \ldots$ & 44844.80 & +073230.1 & 10.58 & 0.55 & 0.06 & 36 & 2.8 & $\mathrm{~A} 2$ \\
\hline $00684.01112 \ldots \ldots \ldots$ & 44858.57 & +072946.2 & 11.58 & 0.44 & 0.07 & 25.9 & 4.4 & A2 \\
\hline $00684.01148 \ldots \ldots$. & 44926.55 & +072934.6 & 10.91 & 0.64 & 0.06 & 33 & 2.7 & A2 \\
\hline $00684.00940 \ldots \ldots$. & 45153.52 & +084641.0 & 11.37 & 0.27 & 0.06 & 75.1 & 6.2 & A3 \\
\hline $00684.00488 \ldots \ldots \ldots$ & 45213.32 & +083630.8 & 11.85 & 0.97 & 0.09 & 88.3 & 2.6 & A3 \\
\hline $00684.01221 \ldots \ldots \ldots$ & 45225.71 & +085536.9 & 11.47 & 0.53 & 0.10 & 60.9 & 5.5 & A3 \\
\hline $00684.01298 \ldots \ldots$. & 45252.06 & +084201.5 & 11.25 & 0.37 & 0.06 & 21.3 & 4.3 & A3 \\
\hline $00684.00509 \ldots \ldots$. & 45258.91 & +083840.4 & 10.97 & 1.94 & 0.12 & 13.1 & 1.8 & A3 \\
\hline $00684.00844 \ldots \ldots$. & 45300.12 & +083036.7 & 11.06 & 0.24 & 0.05 & 88.4 & 5.8 & A3 \\
\hline $00684.00592 \ldots \ldots \ldots$ & 45320.16 & +084326.3 & 11.32 & 0.38 & 0.05 & 47.3 & 3.6 & $\mathrm{~A} 3$ \\
\hline $00684.00849 \ldots \ldots$ & 45331.32 & +084923.2 & 11.31 & 1.33 & 0.07 & 12.0 & 1.4 & A3 \\
\hline $00684.00863 \ldots \ldots$ & 45414.48 & +083033.6 & 10.65 & 0.44 & 0.07 & 78.0 & 4.3 & A3 \\
\hline $00684.00527 \ldots \ldots$. & 45423.56 & +083317.7 & 11.13 & 0.56 & 0.06 & 71.4 & 3.1 & A3 \\
\hline $00688.00926 \ldots \ldots$ & 45309.18 & +095843.3 & 11.98 & 0.75 & 0.05 & 12.2 & 2.0 & A4 \\
\hline $00688.01272 \ldots \ldots \ldots$ & 45326.84 & +094558.1 & 11.02 & 0.79 & 0.06 & 60.6 & 2.2 & A4 \\
\hline $00688.00148 \ldots \ldots$. & 45330.18 & +10 1837.4 & 12.83 & 1.04 & 0.07 & 25.3 & 2 & A4 \\
\hline $00688.01663 \ldots \ldots$. & 45347.45 & +094719.1 & 12.34 & 0.65 & 0.14 & 56.5 & 6.0 & A4 \\
\hline $00688.01528^{\mathrm{d}} \ldots \ldots$ & 45354.69 & +100742.2 & 9.68 & 0.48 & 0.06 & 73.3 & 3.8 & A4 \\
\hline $00688.01652 \ldots \ldots$ & 45425.24 & +093627.2 & 10.09 & 0.63 & 0.07 & 76.3 & 3.1 & A4 \\
\hline $00688.00628 \ldots \ldots$. & 45427.74 & +094847.7 & 10.01 & 0.67 & 0.03 & 49.8 & 1.3 & A4 \\
\hline $00688.01092 \ldots \ldots \ldots$ & 45430.32 & +095803.5 & 10.88 & 0.94 & 0.07 & 65.6 & 2.1 & A4 \\
\hline $00688.00866 \ldots \ldots$ & 45433.71 & +094145.9 & 11.66 & 0.88 & 0.10 & 65.9 & 3.3 & A4 \\
\hline $00688.01497 \ldots \ldots$. & 45523.48 & +101837.1 & 12.70 & 1.12 & 0.06 & 11.4 & 1.5 & A4 \\
\hline $00688.01209 \ldots \ldots \ldots$ & 45523.15 & +095336.6 & 11.71 & 0.50 & 0.05 & 43.6 & 2.9 & A4 \\
\hline $00688.01456 \ldots \ldots$ & 45524.76 & +094657.2 & 12.24 & 1.03 & 0.07 & 64.1 & 1.9 & A4 \\
\hline $00688.01128 \ldots \ldots$. & 45525.50 & +095116.0 & 11.28 & 0.80 & 0.06 & 67 & 2.1 & A4 \\
\hline $00688.01848 \ldots \ldots$. & 45532.75 & +094255.7 & 10.05 & 0.54 & 0.05 & 51.1 & 2.4 & A4 \\
\hline
\end{tabular}

${ }^{a}$ HD 15366.

b SAO 110857.

${ }^{c}$ HD 287164.

${ }^{d}$ HD 287323. 


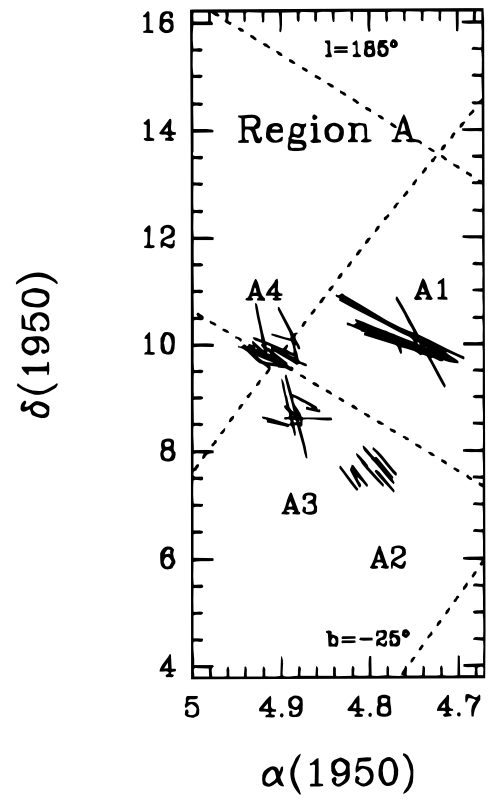

FIG. $2 a$

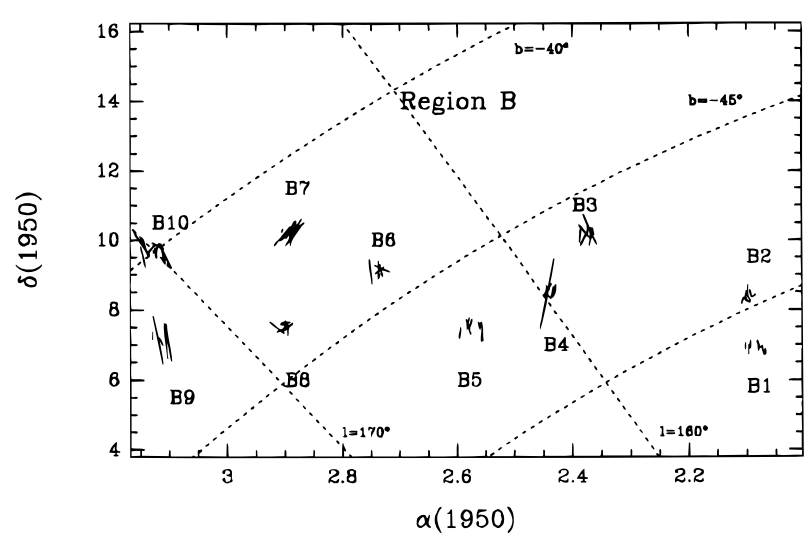

FIG. $2 b$

FIG. 2.-Polarization vectors in the area mapped. (a) region A and (b) region B. The scale is the same as in Fig. 1.

exposure times for program stars ranged between 5 and 30 minutes.

The polarization vectors are shown in Figure 2. At very high latitudes (fields B1-B3) the polarization is $\sim 0.20 \%$. Even in the small polarization fields (B1-B3), there is a good alignment of the polarization vectors. The distribution of the error in the position angle, $\sigma(\theta)$, versus the percent polarization, $P(\%)$, is displayed in Figure 3. As expected, the stars with the largest errors in the position angle are those with the smallest polarization.

The fields we observed are not far from the ecliptic: the B region goes from ecliptic latitude $<-3^{\circ}$ to $<-10^{\circ}$ and region $\mathrm{A}$ is at $<-10^{\circ}$. The $I R A S$ maps at $100 \mu \mathrm{m}$ were corrected for the zodiacal light, but such a correction is not necessary for the polarization data. The polarization of the zodiacal dust decreases rapidly as a function of ecliptic lati-

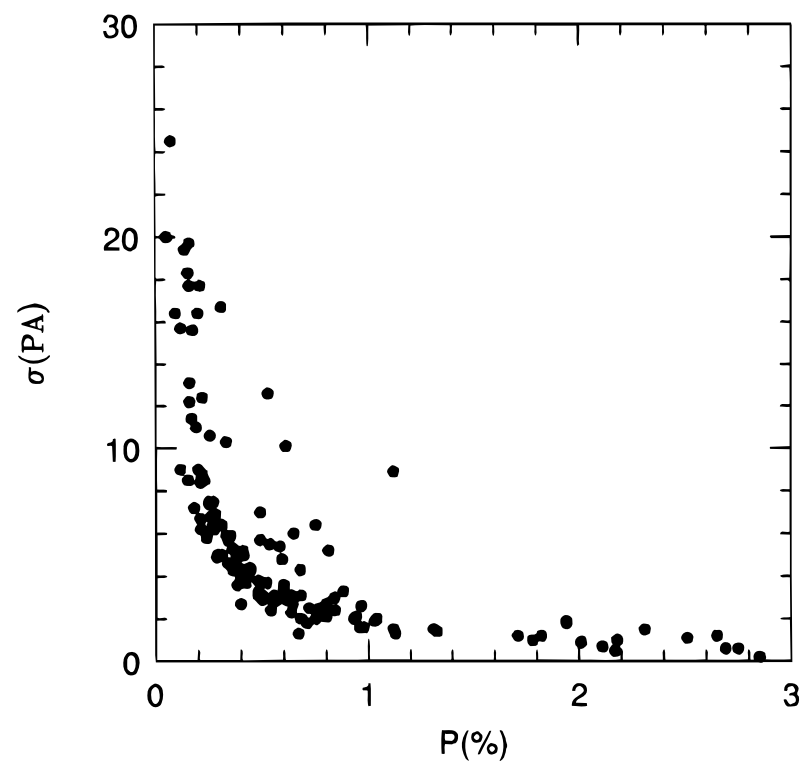

FIG. 3.-Error on the position angles, $\sigma$ (P.A.), vs. $P$ tude (Levasseur-Regourd, Renard, \& Dumont 1990) and is due to the scattering of solar light by these particles. The dust grains near the ecliptic plane have rather heterogeneous scattering properties so that they are unlikely to mimic the polarization of background stellar light by dichroic extinction, as is the case for interstellar polarization.

\subsubsection{Region $A$}

There is a broad distribution in position angle (hereafter P.A., or $\theta)$ from $\theta=0^{\circ}$ to $90^{\circ}$ at low polarization $(P \leq 0.4 \%)$ (see histogram in Fig. 4). There are several data points with $P \geq 0.6 \%$ with similar P.A.s. They appear in the fields A1 and A2 as shown in Figure 5, where the percent polarization as a function of the P.A. is represented for each individual field. The mean $I R A S$ flux, P.A. and $P(\%)$ is

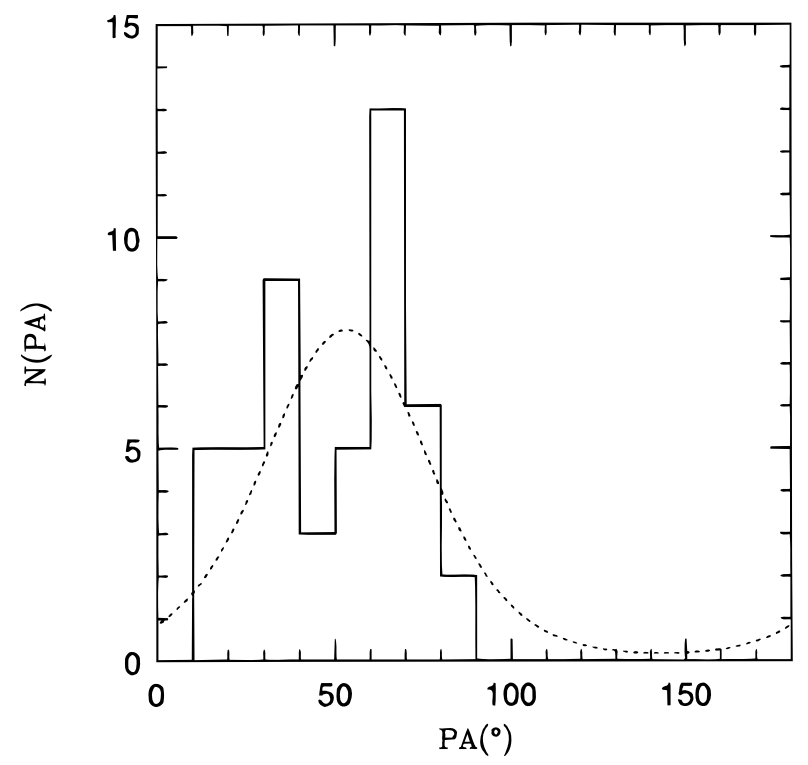

FIG. 4.-Histogram of the position angles for the targets in region A. The curve shows a fit to the dispersion in position angles using the models of Myers \& Goodman (1991). 


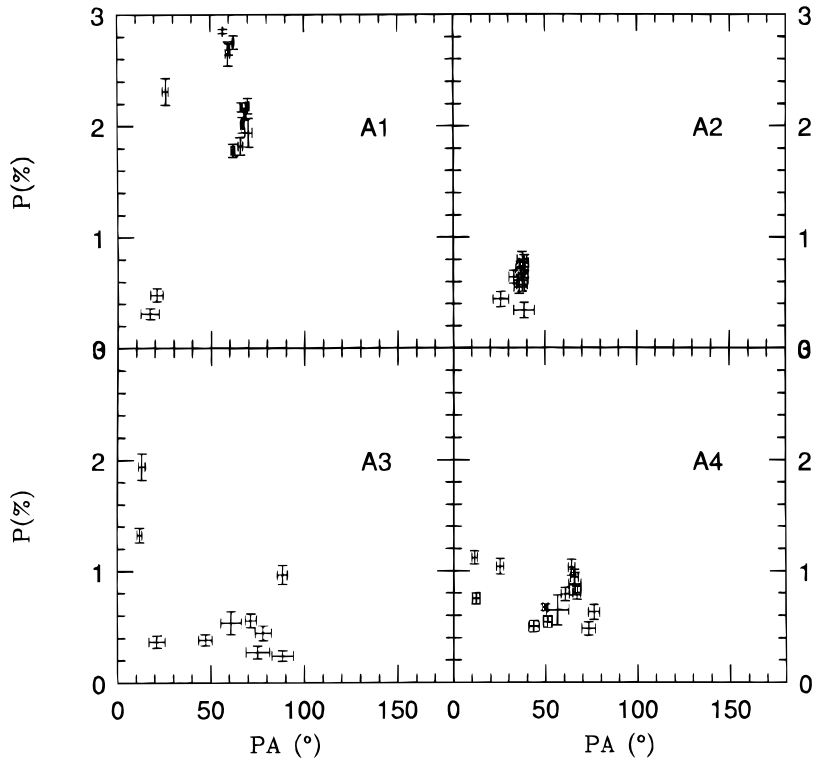

Fig. 5.-Polarization vs. position angle for the four fields in region A

summarized in Table 2 for each field. Because there are two concentrations of values of $P$, there are at least two clumps of polarizing dust along the line of sight toward region A.

The highly polarized field A1 has a mean polarization of $\simeq 2.0 \pm 0.8 \%$. The $100 \mu \mathrm{m}$ flux toward the targets in this field is $18.8 \pm 0.7 \mathrm{MJy} \mathrm{sr}^{-1}$, the highest in our survey (see Fig. 1). The P.A. of the polarization vectors in the concentration is $\theta=55^{\circ}$ with a dispersion of $19^{\circ}$. This orientation is parallel to the large-scale distribution of the emitting dust (see Fig. 1); therefore the local magnetic field is aligned with the dust lane.

The field A2 traces the more diffuse medium in region A with just $7.3 \mathrm{MJy} \mathrm{sr}^{-1}$ of flux at $100 \mu \mathrm{m}$. The dispersion in the P.A. of the polarization vector is only $4^{\circ}$ although the percent polarization is significantly lower than in A1, just $0.6 \%$. The fields $\mathrm{A} 3$ and $\mathrm{A} 4$ are more confusing with a not so well-defined P.A. and intermediate $I R A S$ fluxes (see Table 2). Also they are located in transition regions between dense dust clouds and the diffuse cirrus (see Fig. 1a). There is no obvious relation between the IRAS cirrus morphology and the magnetic field orientation as derived from the P.A. of the polarization vectors in these three more diffuse regions.

\subsubsection{Region $B$}

The mean values per field of the percent polarization, position angle of the polarization vector, IRAS $100 \mu \mathrm{m}$ flux, and magnitude are given in Table 2 as for region $A$. The P.A. is typically between $150^{\circ}$ and $200^{\circ}$ (see Fig. 6). The examination of the individual fields indicates that the P.A. is well defined in the fields: B1, B5, B7, and B9 (see also Fig. 7). This good alignment is remarkable for $\mathrm{B} 1$ since the percent polarization is very low $P(\%)=0.24 \pm 0.05$ and the ISM very diffuse (the $I R A S$ flux at $100 \mu \mathrm{m}$ is just $4.1 \mathrm{MJy} \mathrm{sr}^{-1}$ ).

The field B10 samples the southern border of the HLMC, MBM 16. This cloud is at a distance between 60 and 95 pc (Hobbs et al. 1988). The high polarization indicates that our stars are behind the cloud and sets up a lower limit to the distance of our survey of $\sim 100 \mathrm{pc}$. Other arguments concerning the distance are discussed below (§ 4.1). MBM 16 is a filamentary molecular cloud elongated in the east-west direction. Our data show that the direction of the magnetic

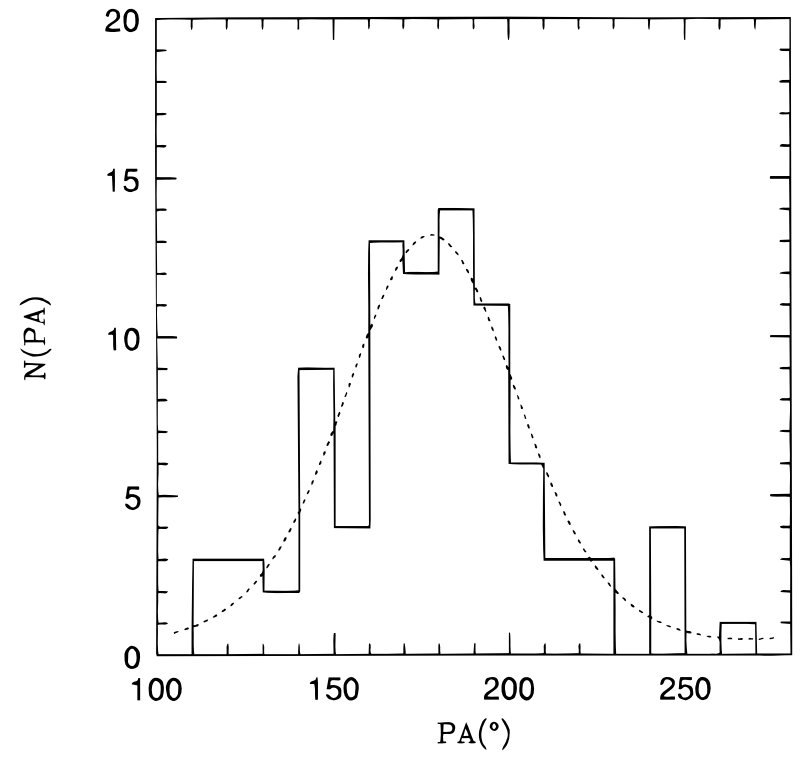

FIG. 6.- Histogram of position angles for the targets in region B

TABLE 2

Mean Values Per Field ${ }^{a}$

\begin{tabular}{|c|c|c|c|c|c|c|c|c|c|}
\hline Field & $P(\%)$ & $\sigma(P)$ & $\theta$ & $\sigma(\theta)$ & $\begin{array}{c}F_{100} \\
\left(\mathrm{MJy} \mathrm{sr}^{-1}\right)\end{array}$ & $\sigma\left(F_{100}\right)$ & Magnitude & $\sigma(\mathrm{Mag})$ & $N^{\mathrm{b}}$ \\
\hline $\mathrm{A} 1 \ldots \ldots$ & 2.0 & 0.8 & 55 & 19 & 18.8 & 0.7 & 11.5 & 1 & 14 \\
\hline A $2 \ldots \ldots$ & 0.6 & 0.1 & 36 & 4 & 7.3 & 0.3 & 11.5 & 0.6 & 10 \\
\hline A $3 \ldots \ldots$ & 0.7 & 0.6 & 55 & 30 & 9.1 & 0.3 & 11.3 & 0.3 & 10 \\
\hline A4 $\ldots \ldots \ldots$ & 0.8 & 0.2 & 52 & 21 & 14.7 & 2.3 & 11.3 & 1 & 14 \\
\hline B1 ....... & 0.24 & 0.05 & 7 & 10 & 4.1 & 0.1 & 11.8 & 0.6 & 8 \\
\hline B2 ........ & 0.17 & 0.06 & 6 & 39 & 5.6 & 0.4 & 12.2 & 1.3 & 8 \\
\hline B3 ........ & 0.44 & 0.28 & 11 & 24 & 6.8 & 0.5 & 12.2 & 1 & 10 \\
\hline B4 ....... & 0.51 & 0.71 & 168 & 23 & 4.9 & 0.2 & 11.7 & 1.6 & 10 \\
\hline B5 ........ & 0.37 & 0.15 & 179 & 10 & 7.0 & 1.0 & 11.6 & 0.8 & 8 \\
\hline B6 ....... & 0.48 & 0.23 & 2 & 39 & 9.3 & 1.9 & 12.3 & 0.9 & 6 \\
\hline B7 ........ & 0.61 & 0.27 & 151 & 14 & 14.1 & 1.9 & 12.7 & 1 & 11 \\
\hline B8 ........ & 0.32 & 0.13 & 169 & 56 & 11.6 & 1.5 & 12.2 & 1.4 & 9 \\
\hline B9 $\ldots \ldots \ldots$ & 0.91 & 0.59 & 10 & 8.8 & 14.4 & 2.3 & 11.8 & 0.25 & 6 \\
\hline $\mathrm{B} 10 \ldots \ldots$ & 0.58 & 0.26 & 11 & 28 & 14.9 & 1.4 & 12.0 & 1.5 & 12 \\
\hline
\end{tabular}

${ }^{\text {a }} \sigma$ values are standard deviations from the mean for each field and physical magnitude.

${ }^{b}$ Number of stars in each field. 


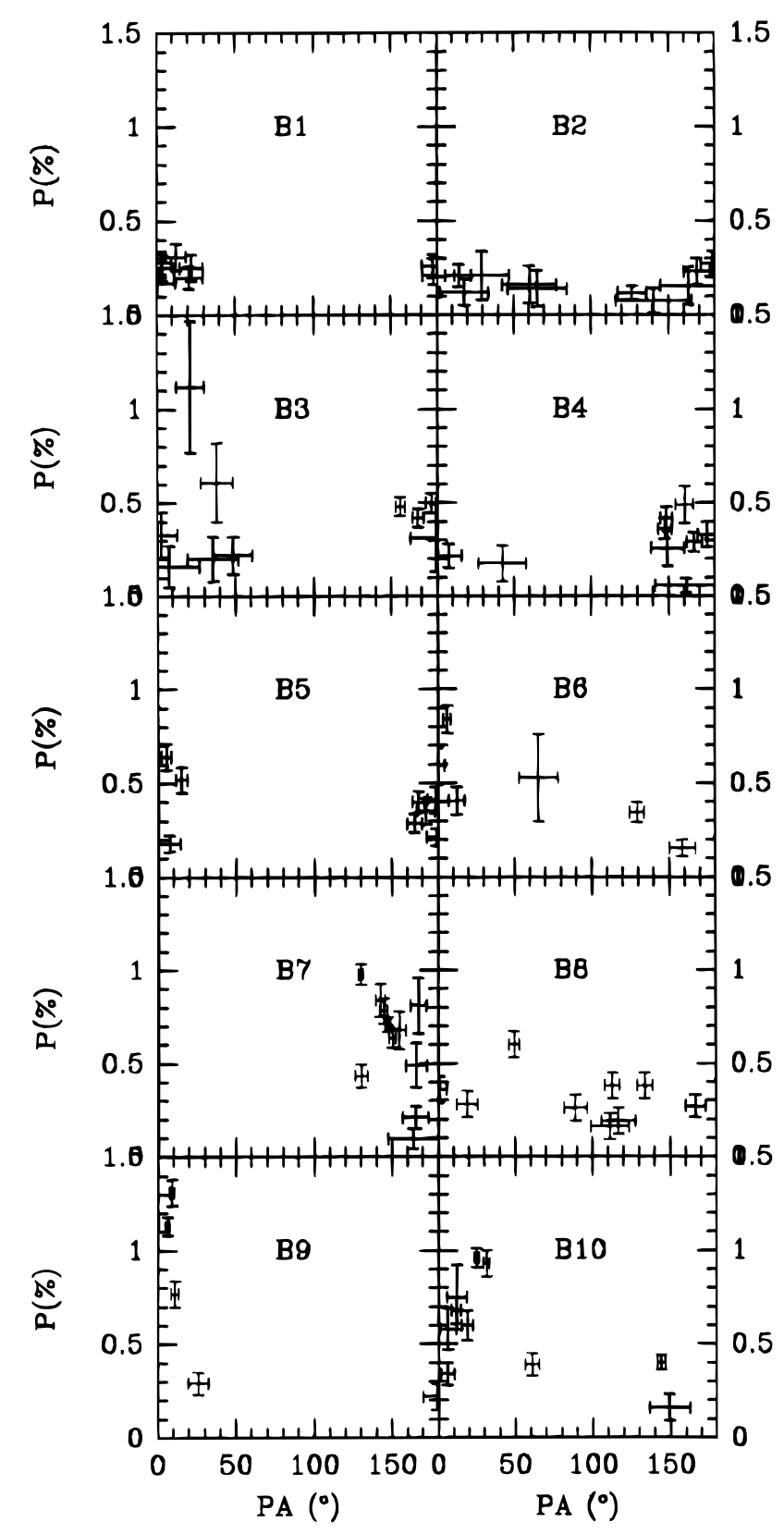

FIG. 7.-Polarization vs. position angle for the 10 fields in region $B$

field is perpendicular to this filament of molecular gas. A similar orientation of the field with respect to the cloud elongation has been observed in other molecular clouds such as B216/217 (Heyer et al. 1987), Lupus I (Strom, Strom, \& Edwards 1988), and L204 (McCutcheon et al. 1986). We refer the reader to Bastien (1991) for other clouds with different field orientations.

\section{ANALYSIS}

In this section, we compare our measured polarization values with the properties of the local interstellar medium (LISM) and the mean galactic model for the $\mathrm{H}$ I distribution, in order to more clearly identify the significant magnetic field structure at high latitudes. Then, we account for the measured values of the polarization of our targets using the IRAS data.

\subsection{The LISM: Clumps}

The ISM around the Sun is clumpy; $80 \%$ of the mass is in clumps of cold $\mathrm{H}$ I that fill only $10 \%$ of the volume (Trapero et al. 1992). The Sun is surrounded by a local cloud of radius $2 \mathrm{pc}$ (Frisch 1981) and particle density $n=0.1 \mathrm{~cm}^{-3}$ whose detection would require much larger sensitivities than that obtained with our instrumental setup. Farther out, the Sun is within the local bubble of hot ionized medium (HIM) at $T=10^{6} \mathrm{~K}$. The size of the bubble has been determined from its X-ray emission; it has an equivalent radius of $150-200 \mathrm{pc}$ toward the south galactic plane and galactic longitudes between $180^{\circ}$ and $250^{\circ}$ (Snowden et al. 1990). This is the area sampled in our survey. The topology of the HIM is unclear but it is best explained by the McKee \& Ostriker (1977) model. In this model hot gas produced in supernova explosions fills the ISM surrounding clumps of cold neutral medium (CNM) until they evaporate. The typical density and size of these local clumps are $n=40 \mathrm{~cm}^{-3}$ and $R=2 \mathrm{pc}$. The expected contribution of one of these clumps to the polarization can be derived from the well-known relation between the $\mathrm{H}$ I column density in the line of sight to various stars, and the color excess $E(B-V)$ which measures the total amount of dust in that line of sight:

$$
N(\mathrm{H})=5.8 \times 10^{21} E(B-V) \mathrm{cm}^{-2}
$$

(e.g., Spitzer 1978).

The classic paper by Serkowski, Mathewson, \& Ford (1975, henceforth SMF) established that interstellar polarization takes its maximum value at a wavelength of $\lambda_{\max } \simeq$ $0.45 \mu \mathrm{m}-0.8 \mu \mathrm{m}$. This quantity can be determined observationally for each star. For the large sample of stars studied by SMF, the median value is less than $\lambda_{\max }>=0.55$ $\mu \mathrm{m}$. It is proportional to the average size of the grains on the line of sight to each star.

More extensive recent investigations have generalized the SMF relation to the empirical formula (Whittet et al. 1992)

$$
\frac{p(\lambda)}{p_{\max }}=\exp \left[-K \ln ^{2}\left(\frac{\lambda_{\max }}{\lambda}\right)\right] \equiv \alpha
$$

and

$$
K=0.01+1.66 \lambda_{\max },
$$

where $p(\lambda)$ is the percent polarization at a wavelength $\lambda$, and $p_{\max }$ is the peak polarization occurring at $\lambda_{\max }$. The maximum polarization correlates well with $E(B-V)$ :

$$
p_{\max } \leq 9.0 E(B-V) \text {. }
$$

For our observations, the effective wavelength was $\lambda \simeq 0.7625 \mu \mathrm{m}$. Thus, using the median value of $\lambda_{\max }$ of 0.55 $\mu \mathrm{m}$ in equation (3.2) we obtain the median value; $\langle\alpha\rangle=0.91$.

The link between polarization and column density is obtained on combining the relations (3.1)-(3.3);

$$
P(\lambda=0.7625 \mu \mathrm{m}) \leq \frac{\alpha}{6.4} N_{20} \mathrm{~cm}^{-2}=0.14 N_{20} \mathrm{~cm}^{-2},
$$

where the $\mathrm{H}$ I column density is measured in units of $10^{20}$ $\mathrm{cm}^{-2}$. Therefore a clump with a column density characteristic of the LISM clumps, that is, $N_{20}=5 \mathrm{~cm}^{-2}$, produces a 
maximum polarization of $0.7 \%$ which can be easily detected. It is clear from Figures 5 and 7 that we are generally measuring a more diffuse medium since only occasionally do we detect sudden increments of polarization in our fields. Moreover, since the interstellar clouds can be traced either in polarization or through thermal emission, these LISM clumps also show up in the IRAS maps that therefore can be used to trace the clumpiness in the ISM (see below).

\subsection{Model for Galactic $\mathrm{H}$ I}

The $\mathrm{H}$ I layer has a vertical extent not much greater than $1 \mathrm{kpc}$ and there are many irregularities in its lower regions (Lockman, Hobbs, \& Shull 1986 and references therein). The mean $\mathrm{H}$ I column density for gas with galactic latitude $|b| \geq 2.5$ is well fitted by the expression (see DL),

$$
\bar{N}(\mathrm{H})=2.9 \times 10^{20} \mathrm{csc}|b| \pm 1.4 \times 10^{20} \mathrm{~cm}^{-2} .
$$

If the galaxy consisted of a uniform gas layer, then one would expect that $N_{\mathrm{H}} \sin b=$ const. Plots of $N_{\mathrm{H}}|\sin b|$ versus $|\sin b|$ show, however, that there is a large dispersion, and regions of excess value often correspond to the filamentary structure.

The behavior of the mean gas density as a function of scale height $z$ is also given by DL. They suggest that for galactic radii between 4 and $8 \mathrm{kpc}$, the best estimate for $n(z)$ consists of two Gaussians (central densities, 0.395 and 0.107 $\mathrm{cm}^{-3}$; FWHMs, 212 and $530 \mathrm{pc}$ ) and an exponential (central density, $0.064 \mathrm{~cm}^{-3}$; scale height, $403 \mathrm{pc}$ ). We have computed the total column density predicted by this model and obtain

$$
\bar{N}_{20}|\sin b|=3.12 \text {. }
$$

\subsection{IRAS Fluxes}

Several studies of high-latitude clouds have shown that the far-IR emission of low-velocity $\mathrm{H}$ I clouds at high latitude correlates well with the extinction and $\mathrm{H}$ I column density (Boulanger, Baud, \& van Albada 1985; De Vries \& Le Poole 1985; Wakker \& Boulanger 1986; for low latitudes, see Terebey \& Fich 1986). Thus, for a specific field at $b=+60^{\circ}$, Martin et al. (1994) find the relation

$$
F_{100}=0.85 N_{20}-1.4,
$$

where $F_{100}$ is the IRAS $100 \mu \mathrm{m}$ flux measured in units of $\mathrm{MJy} \mathrm{sr}^{-1}$. A larger scale average of high-latitude IRAS emission was compared with $\mathrm{H}$ I maps by Boulanger \& Perault (1988, henceforth BP). These authors found that a linear, least-squares fit to the $100 \mu \mathrm{m}$ profiles at $b<-20^{\circ}$ gives the cosecant law

$$
\bar{F}_{100}=\frac{3.4}{|\sin b|}-1.7 .
$$

This is a more appropriate form for our purposes because our data span such a large portion of the high-latitude sky along the anticenter stream.

Using the mean galactic $\mathrm{H}$ I model (eq. [3.6]) to eliminate $|\sin b|$ in equation (3.7b), one finds that the IRAS flux associated with the smooth $\mathrm{H}$ I component is

$$
\bar{F}_{100}=1.1 \bar{N}_{20}-1.7 \text {. }
$$

We plot the relation between $F_{100}|\sin b|$ and $|\sin b|$ toward our target stars in Figure 8. Also shown in Figure 8

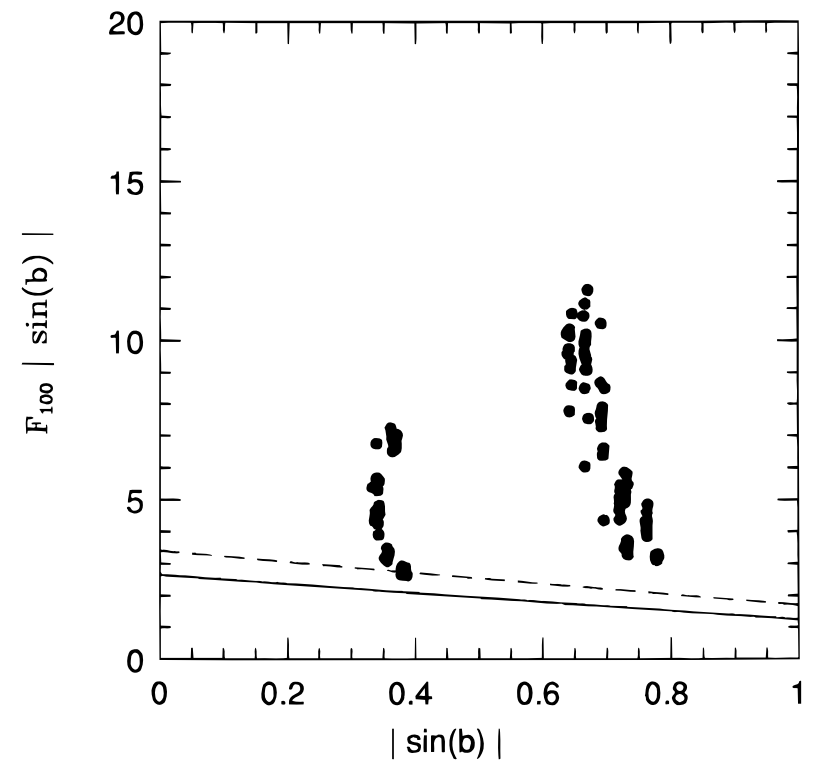

Fig. 8. $-F_{100}|\sin b|$ vs. $|\sin b|$. The dashed and continuous lines represent the expected correlations derived from the DL mean galactic model and the eqs. $3.7 \mathrm{a}$ and $3.7 \mathrm{~b}$, respectively.

is the predicted relation for the mean galactic gas model, prescribed by equations (3.7a) and (3.7b) for the models by Martin et al. and BP, respectively. The data points that lie significantly above these lines are highly significant; these are structures that stand out strongly against the mean $\mathrm{H} \mathrm{I}$ of the Galaxy. They represent either diffuse cirrus or molecular clouds detected by $I R A S$ and have fluxes $\geq 3-5$ MJy $\mathrm{sr}^{-1}$ producing a measurable polarization that can be detected in our survey.

\subsection{Polarization}

In Figure 9 we plot the percent polarization versus the IRAS flux toward our targets. Only data with a small relative error in $P(\%), \sigma(P) / P \leq 0.3$, are shown. This corresponds to $\sigma(\theta) \leq 8.6$. There is a clear-cut correlation between the percent polarization and the IRAS flux:

$$
P(\%) \leq(0.16 \pm 0.05) F_{100} .
$$

If we substitute $F_{100}$ using equation $3.7 \mathrm{a}$, then

$$
P(\%) \leq(0.13 \pm 0.03) N_{20}-0.22,
$$

which is very similar to equation (3.4) apart from a constant term. The intercept probably varies due to the lack of an absolute calibration of the IRAS data itself. Two of the stars shown in Figure 9, GSC 00632.01103 and GSC 00684.00509, have an abnormally high polarization even though the P.A.s of their polarization vectors are similar to those of other stars in the same fields. This suggests that their polarization is not intrinsic. They could probably be distant sources, or sources that lie behind very small clumps of unusually high column density that are not resolved by $I R A S$ (the $100 \mu \mathrm{m} I R A S$ maps have a resolution of 1.5).

We conclude that the column of warm dust detected at $100 \mu \mathrm{m}$ is the same that is responsible for the polarization of the stellar radiation. Thus, our polarization measurements trace the magnetic field geometry in the cirrus clouds detected in the $100 \mu \mathrm{m} I R A S$ band. Note that in the regions of low fluxes $\left(F_{100} \leq 8 \mathrm{MJy} \mathrm{sr}^{-1}\right)$ and of high fluxes 


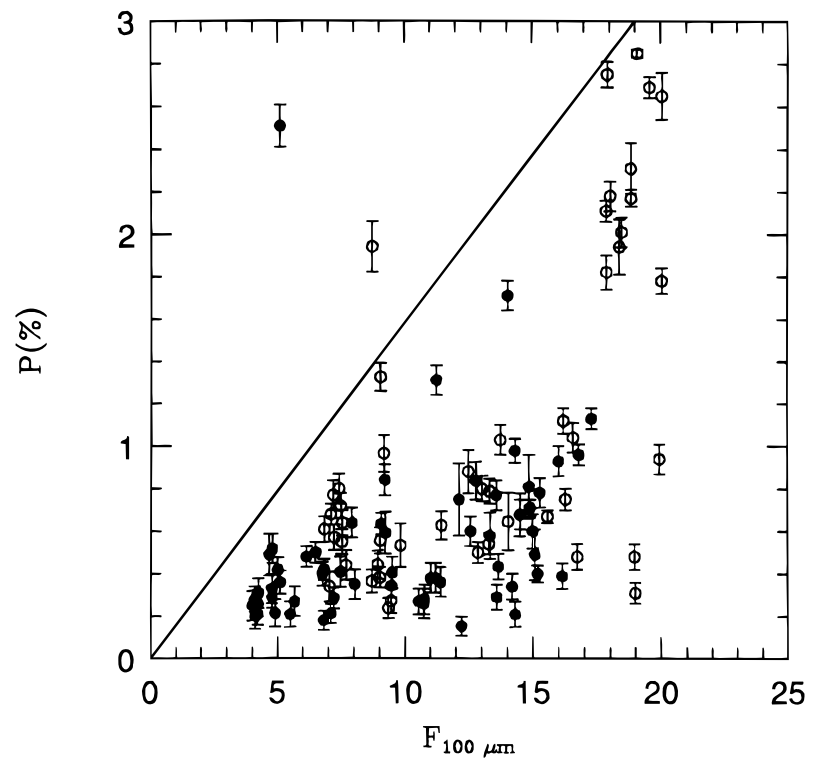

Fig. 9.-Polarization, $P(\%)$, vs. infrared flux, $F_{100}$. Open and filled circles correspond to sources in regions $\mathrm{A}$ and $\mathrm{B}$, respectively. Only points with relative error in $P(\%)$ smaller than 0.3 are plotted. The straight line represents the correlation derived from our data (see text) and presents a very good upper envelope to the data. The two stars above the line are also discussed in the text.

$\left(F_{100} \geq 16 \mathrm{MJy} \mathrm{sr}^{-1}\right)$ there are stars with polarization values as high as expected if all the dust grains emitting at $100 \mu \mathrm{m}$ were also involved in the polarization of the stellar light (see Fig. 9). This indicates that our survey is as deep as the IRAS survey in these regions. The high flux limit corresponds to region A (field A1) in the outskirts of the OrionMonoceros complex at a distance of 500-700 pc. The low flux limit corresponds to diffuse IR $A S$ cirrus in the fields A2, B1-B5. Fluxes $\left(F_{100}\right)$ between 8 and $16 \mathrm{MJy} \mathrm{sr}^{-1}$ are detected in the lines of sight toward sources having polarization values significantly lower than that implied by their IRAS fluxes. This suggests that the magnetic field orientation changes significantly with the column density in this area. This is further confirmed in Figure 10 where the P.A. is plotted versus the $F_{100}$ for the same sources. Note that the dispersion in P.A. is significantly larger for these intermediate IRAS fluxes. They correspond to the fields B7-B10 in region $\mathrm{B}$ and $\mathrm{A} 3-\mathrm{A} 4$ in region $\mathrm{A}$.

\subsection{Polarization Position Angles}

We have performed fits to the Gaussian-like distributions of position angles for regions A and B separately (Figs. 4 and 6 , respectively). We followed the method developed by Myers \& Goodman (1991). The results are given in Table 3. The columns give respectively the name of the region, the total number of stars included in the fit, the mean position angle and its error, the dispersion of the distributions and

TABLE 3

Distribution of Polarization POSITION ANGLES

\begin{tabular}{lcrc}
\hline \hline Region & $N^{\mathrm{a}}$ & $\Theta_{\boldsymbol{E}}$ & $s$ \\
\hline A $\ldots . .$. & 48 & $53^{\circ} \pm 5^{\circ}$ & $0.43 \pm 0.05$ \\
B $\ldots . .$. & 88 & $178^{\circ} \pm 4^{\circ}$ & $0.47 \pm 0.08$ \\
\hline
\end{tabular}

${ }^{a}$ Number of stars in each region.

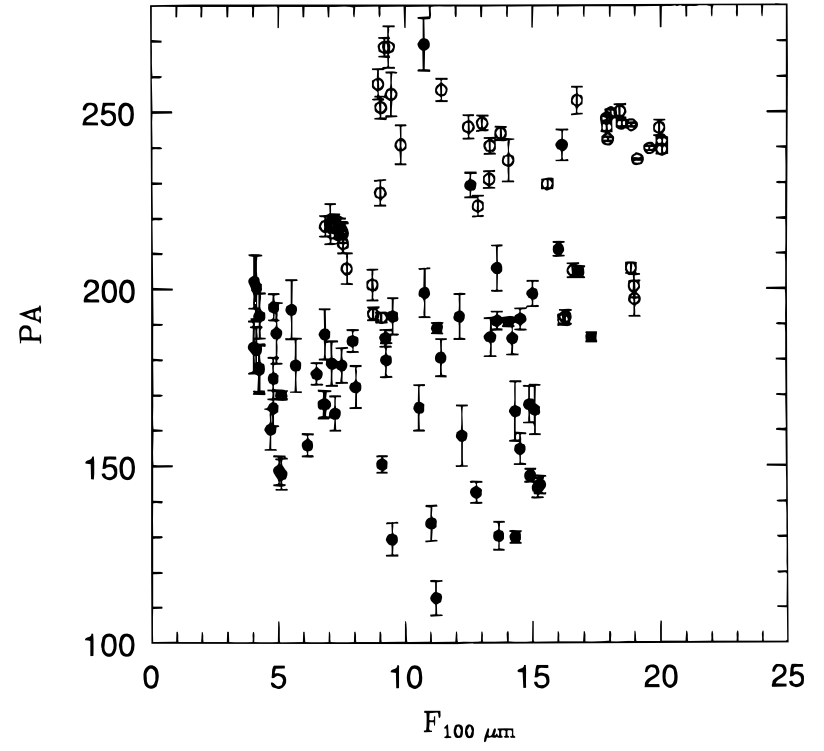

FIG. 10.-Position angles vs. infrared flux, $F_{100}$. Open and filled circles correspond to sources in regions A and B, respectively. Only points with relative error in $P(\%)$ smaller than 0.3 are plotted.

its error. The errors are based on nonlinear least-squares fits of the $f^{3 \mathrm{D}}$ model of Myers \& Goodman. The distribution of position angles for both regions has a single maximum, with dispersion of around 0.4 radians. Such values are typical of the values found for clouds with embedded clusters, whereas clouds without clusters usually have dispersions of $0.2-0.3$. According to their model, the field can be divided into uniform and nonuniform components. The nonumiform component has a certain number of correlation lengths along the line of sight through the cloud. Typical values which have been evaluated by Myers \& Goodman (1991) are of order a few. For example, for L204, they obtained 3 or 4 according to whether the absorption or emission measurements of the $\mathrm{H}$ I Zeeman observations were used for the calculation, respectively. The number of correlation lengths cannot be determined for our two regions since Zeeman observations are not available, but we expect that it should be comparable.

\section{DISCUSSION}

\subsection{High-Velocity Clouds}

If the ACHVCs are within several hundred pc (Mirabel 1982; Giovanelli 1980b), there is a chance that some stars in our sample are behind the stream. One way to get an estimate of the distance to the clouds is to find the distance to our program stars. Four of the brightest stars in our sample appear in the SIMBAD database, and one of those stars has a known distance. It is listed as a probable nonmember of the Hyades star cluster by Schwan (1991). The distance that he found is $69.3 \mathrm{pc}$ for SAO $110857\left(=\mathrm{BD}+07^{\circ} 451=\right.$ 0641.00285). This star is in region B8 (see Table 1), and this region is characterized by a large dispersion in position angles, as shown in Table 2 . Therefore, it cannot be readily related to the other stars in the sample, and cannot be used to put a reliable constraint on the distance to the HVCs. Tamanaha (1996) has recently put constraints on some of the anticenter HVCs. He studied four regions in particular. One of them, "Cohen's stream." covers the southern part of our region B, and is located at more than $350 \mathrm{pc}$. Another 
region, AC I, located about $10^{\circ}$ northeast of our region $\mathrm{A}$, is at more than $280 \mathrm{pc}$, according to him. These results are consistent with our analysis in $\S 3$ above.

The precise location of the anticenter HVCs in the sky is plotted in Figure 11 using Giovanelli's (1980a) data. He rastered this area with scans of constant declination at 0 , $2.5,5,7.5,8.75,10,12.5$, and 15 degrees. The complex is between $7.5 \leq \delta \leq 10^{\circ}$ and the two regions $\mathrm{A}$ and $\mathrm{B}$ are clearly identifiable in the figure. The stream extends in the east-west direction (P.A. $=90^{\circ}$ ). The open circles correspond to positions with column densities greater than $1 \times 10^{19} \mathrm{~cm}^{-2}$. The total column density of high-velocity material is very low in most directions. The locations where the column density is high enough to produce a polarization greater than $0.1 \%$ (according to eq. [3.4]) are marked with filled dots. Wakker \& Schwarz (1991) carried out higher resolution mapping of several HVC complexes and observed that the gas is more clumped than the earlier lower resolution surveys suggest. In fact, the peak column densities may increase by a factor 4 depending on the individual cloud. Unfortunately, the ACHVC is not mapped at this improved resolution but we expect that at most the polarization produced by individual clumps reaches $0.40 \%$. This peak value is expected in the fields B4 and B5 of region $\mathrm{B}$ and in A2 of region A. In all of them the dispersion in the P.A. of the polarization vector is small, in particular in A2. The field appears to be perpendicular to the stream especially at high galactic latitudes.

The column density of $\mathrm{H}$ I gas in the high-velocity component is only a few percent of that at the line center. It is therefore impossible to disentangle magnetic structure that is well and truly associated with the HVCs, as compared to the low-velocity gas. We can only safely say that the lowvelocity, high-latitude gas has a well-ordered, associated magnetic field that is perpendicular to the gas filaments. It would be somewhat surprising, however, if the HVCs did not also share the same magnetic field structure in view of their cospatial projected distribution with the low-velocity material and the low dispersion in P.A. along the whole dust column.

\subsection{Magnetic Field Structure at High Latitudes: A Model}

Our observations provide good evidence that the highlatitude clouds that are so well traced out by IRAS have an associated magnetic field. Given the statistical nature of our survey, we feel it safe to say that we have probably probed the galactic magnetic field structure out to several hundred $\mathrm{pc}$ below the plane. The polarization vectors are plotted in galactic coordinates in the top panel of Figure 12. We also plot in the bottom panel, the polarization data of stars in the general field from the survey of Klare \& Neckel (1977). These data complement our own and help to further demarcate the polarization associated with the high-latitude clouds.

Our main finding is that at high latitudes, the galactic magnetic field is perpendicular to the stream. The polarization shows a large $z$-component (perpendicular to the galactic plane) even at latitude $-50^{\circ}$. This is true for clumps such as those detected toward B1 or B5 and also for the more diffuse medium. Only in B7 is the polarization approximately parallel to the galactic plane. Closer to the Galactic plane at latitudes $\geq-25^{\circ}$ the $z$-component is dominant.

A natural explanation for these observations is that the high-latitude $\mathrm{H}$ I filaments are associated with a predominantly toroidal magnetic field. This would explain why the polarization is perpendicular to the $\mathrm{H} \mathrm{I}$. The fact that highspeed gas motion occurs along the filament implies that the toroidal field component is wrapped around the outside of the filament where it cannot impede the infall of the gas. Thus it appears that the magnetic structure of the highlatitude clouds may consist of braided magnetic flux tubes. Gas can fall freely down along the arched magnetic flux filament, back to the Galactic plane as the flux tube rises into the Galactic corona.

Observational support for our hypothesis of predominantly helical field derives from the study of the magnetic field associated with the molecular filament L1641 in the Orion A cloud (see Bally 1987). Both optical polarimetry and Zeeman measurements have been performed which clearly show that a toroidal field is wrapped around the filament. Since the Orion cloud lies near the base of the stream, our interpretation of a predominantly toroidal filament magnetic field seems well justified.

There are good physical reasons why rising magnetic flux tubes should be dominated by a toroidal magnetic field. Magnetic fields in the Galactic plane have energy densities that are roughly in equipartition with that of the gas (e.g., Heiles et al. 1993). As a buoyant magnetic flux tube lifts up out of the Galactic plane and into the low-density Galactic corona, gas will drain out of it and flow back to the midplane of the galaxy (e.g., Matsumoto et al. 1988; Foglizzo \& Tagger 1994). As the flux tube reaches the rarified higher

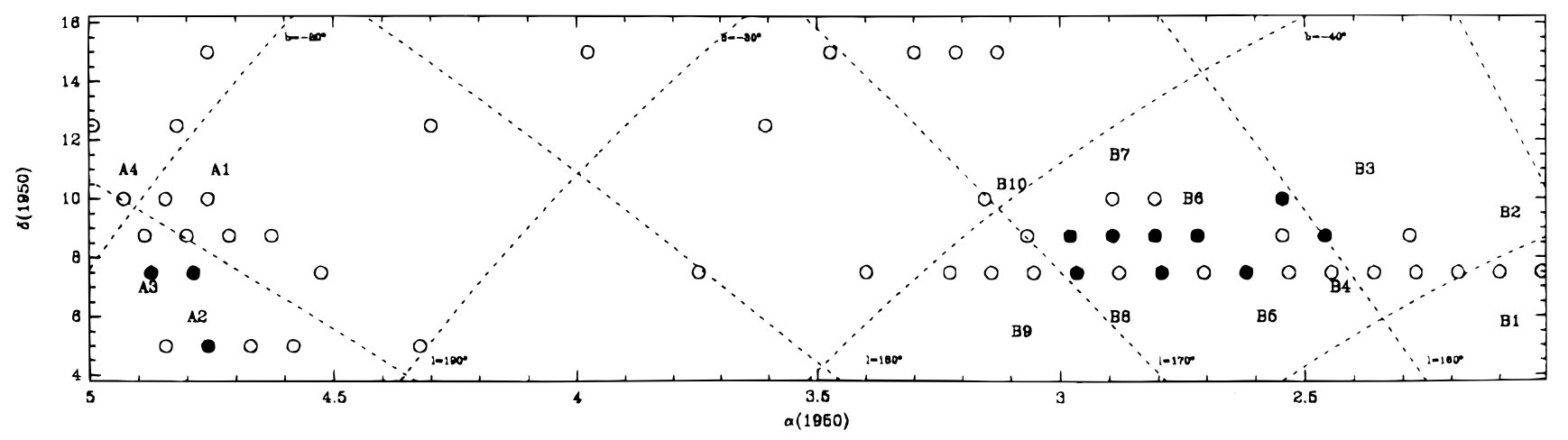

FIG. 11.-Distribution of high-velocity $\mathrm{H}_{\mathrm{I}}$ in the anticenter direction from Giovanelli 1980a. The open circles represent points where the column density is in between $1 \times 10^{19} \mathrm{~cm}^{-2}$ and $1 \times 10^{20} \mathrm{~cm}^{-2}$. The filled circles represent areas with column densities $\geq 1 \times 10^{20} \mathrm{~cm}^{-2}$ and that henceforth would contribute to the total polarization with at least a $0.1 \%$. 

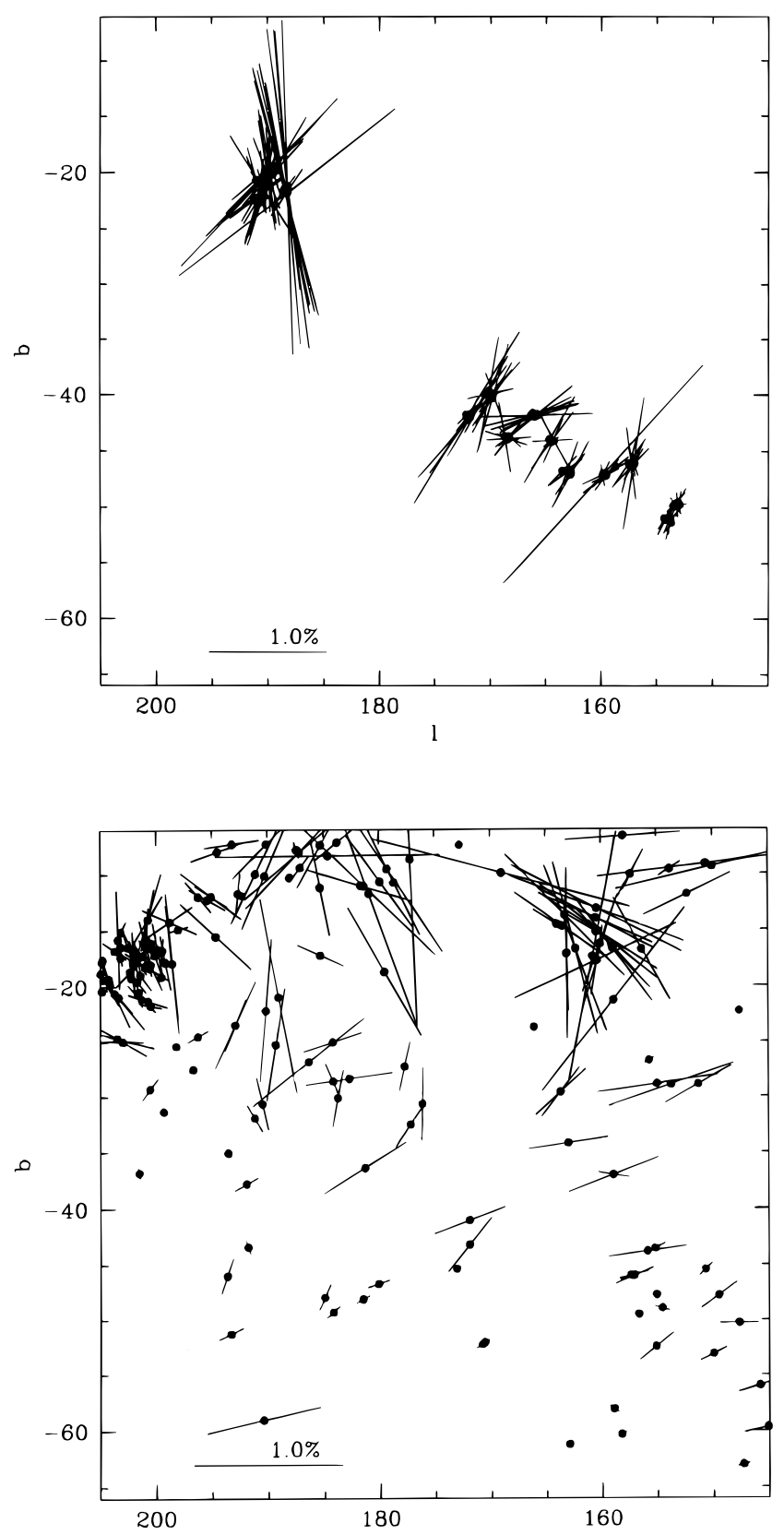

FIG. 12.-Maps with the polarization vectors in galactic coordinates. The upper frame shows the stars in our survey (Table 1), while the lower frame shows stellar polarization data from the Klare \& Neckel (1977) compilation of the general interstellar polarization. There is usually a good agreement between the two data sets. The scale for the linear polarization vectors is indicated at the bottom left of the figure.

latitudes, the magnetic forces begin to exceed any other force. This imbalance of the magnetic force is compensated by a restructuring of the field so that it becomes approximately force-free: the total Lorentz force associated with the flux tube vanishes, $\boldsymbol{J} \times \boldsymbol{B}=0$, where $\boldsymbol{J}$ is the current. It may be shown that for open-ended flux tubes whose end points do not suffer any motion, the fields which minimize the magnetic energy subject to initial and boundary conditions are in general force-free (see Heyvaerts \& Priest 1984, henceforth HP). Thus, the current is parallel to the field, $\boldsymbol{J} \| \boldsymbol{B}$, or

$$
\boldsymbol{\nabla} \times \boldsymbol{B}=\alpha(\boldsymbol{r}, t) \boldsymbol{B},
$$

where $\alpha$ is a scalar function of space and time. A larger value of $\alpha$ corresponds to more current and a stronger twist of the magnetic field line. General solutions of this nonlinear equation are not available. However, HP show that the most relaxed magnetic configurations available are with $\alpha=$ const. The corresponding field configuration therefore obeys the linear vector Helmholtz equation (Chandrasekar \& Kendall 1957).

$$
\nabla^{2} \boldsymbol{B}+\alpha^{2} \boldsymbol{B}=0 .
$$

In the simplest possible theory where the magnetic structure can be represented by a single term in a Bessel series (assume cylindrical coordinates) with corresponding radial wavenumber $k$, one has solutions of the form $B_{r}, B_{\phi} \propto$ $J_{1}(k r) \exp (-\beta z)$ and $B_{z} \propto J_{0}(k r) \exp (-k z)$, where the effective radial wavenumber is $k=\left(\alpha^{2}+\beta^{2}\right)^{1 / 2}$ and where $J_{1}, J_{0}$ are Bessel functions.

The current, in our galactic context, is generated by the relative shear of the two footpoints of the magnetic flux loop, rooted at low Galactic latitudes. This happens for footpoints of field lines that are anchored at slightly different Galactic radii. The resulting relative shear between the footpoints results in a current which generates the toroidal field in the flux rope. Following HP, we suppose that a Galactic magnetic flux loop evolves between states of larger and larger $\alpha$, each of which could be considered to be forcefree. Eventually, the magnetic energy that is stored in this system is released in the form of a flare.

This physical picture is akin to the well studied problem of magnetic flux tubes in the tenuous solar corona (see, e.g., Tandberg-Hanssen \& Emslie 1988). Linear force-free models have, on occasion, been successfully matched to the solar data. Consider the simple model in which a magnetic flux loop is anchored at radii $\pm r_{0}$ (Raadu \& Nakagawa 1971). Then, the twist angle $\gamma$ of the field at the top of the arcade with respect to the axis connecting the two footpoints of the loop is

$$
\frac{\alpha}{\beta}=\tan \gamma .
$$

This result allows $\alpha$ to be found in terms of $\gamma$ and $\beta$ which are given by the observations (the vertical scale of the loop is $H=2 \pi / \beta$ ). As an example, for an observed twist of $60^{\circ}$, say, then $\alpha=2 \pi \tan \gamma / H=10.9 / H$.

Our observation that the dispersion in P.A. is larger in the diffuse medium than in the clumps also appears to have a simple explanation. Consider a turbulent medium in which a magnetic fluctuation has a scale size $\lambda_{B}=2 \pi / k_{B}$. The magnetic field direction remains coherent over a cell (i.e., low dispersion in P.A.), but varies randomly in direction from cell to cell. One can account for our observation if the size of such regions is independent of their surface density (at least for the low to moderate optical depths probed by our survey). For, in this case, when we probe a region of low optical depth, we traverse many scale lengths $\lambda_{B}$ before reaching the surface of unit optical depth. Thus, the net measured polarization, being summed over $N$ randomly oriented cells, would be lower than that of a single cell by a factor of $N^{-1 / 2}$ (where $N$ is the number of cells required to reach an optical depth of unity). Thus the dispersion in P.A. associated with high-density clumps will be much lower than measurements made through the diffuse medium, as our observations demand. The concept of magnetic cells used here is similar to that discussed in $\S 3.5$ above. 


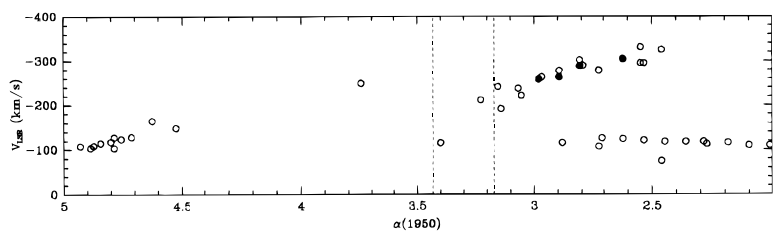

FIG. 13.-Position-velocity diagram of the ACHV and ACVHV streams. The open and filled circles represent areas with column densities as in Fig. 11. The velocity gradient is observed in the east-west direction. The spatial scale is the same than in Figs. $1 a, 1 b, 2 a$, and $2 b$. The location of MBM 16 is marked by dashed lines.

\subsection{Kinematics}

The LSR velocity $\left(V_{\mathrm{LSR}}\right)$ of the ACVHC is plotted versus right ascension in Figure 13 for comparison. The kinematical behavior of the two components: the ACVHV stream and the ACHV stream is very different. The $V_{\mathrm{LSR}}$ of the ACHV stream increases smoothly along its length at a rate of $0.67 \mathrm{~km} \mathrm{~s}^{-1} \mathrm{deg}^{-1}$. However the $V_{\mathrm{LSR}}$ of the ACVHV stream decreases rapidly at a rate of $-11 \mathrm{~km} \mathrm{~s}^{-1} \mathrm{deg}^{-1}$ as it approaches the Galactic plane. The high-latitude molecular cloud, MBM 16, extends between right ascension $3^{\mathrm{h}} 14^{\mathrm{m}}$ and $3^{\mathrm{h}} 26^{\mathrm{m}}$ (see Fig. 13) and it is located close to the region where both streams cross each other in the velocity-position diagram. The IRAS maps also show a shell structure in this area (see Fig. 1) associated with MBM 16. Note that at this location the magnetic field is perpendicular to the stream.

The highest speed that gas could aquire in falling back toward the plane if it started from the top of a loop located at Galactocentric radius $R_{0}$ is just the galactic escape speed, $v_{\text {esc }}\left(R_{0}\right)$. Current measurements indicate that the escape speed in the vicinity of the Sun is

$$
v_{\text {esc }}\left(R_{0}\right)=500 \mathrm{~km} \mathrm{~s}^{-1}
$$

(Binney \& Tremaine 1987). This is more than enough to account for most of the high-velocity clouds.

Finally, we note that according to Blitz, Magnani, \& Wandel (1988), the broad-wing molecular lines measured in MBM 16 do not have an obvious energy source. Note, however, that our observed high-latitude streams intersect at the position of MBM 16. It is possible that the collision of infalling low-density HVCs into this region could provide the needed energy. It remains to be seen whether or not the resulting shocks could explain the details of the spectroscopy of MBM 16.

\section{CONCLUSIONS}

We surveyed the optical polarization of stars in the direction of the anticenter HV and VHV streams in the area $5^{\mathrm{h}} \geq \alpha \geq 2^{\mathrm{h}}$ and $6^{\circ} \leq \delta \leq 12^{\circ}$. We divided this area into two regions (A and B). Region A (Galactic latitude $b \simeq-20^{\circ}$ ) samples the direction toward Orion and region $\mathrm{B}\left(b \simeq-50^{\circ}\right)$, the high-latitude stream. We augmented our data with the $100 \mu \mathrm{m}$ IRAS maps of the region in order to help discriminate polarization that arises from foreground dust from dust that is associated with the high-latitude clouds. The IRAS flux is dominated by the anisotropies of the ISM (shells of warm dust, molecular clouds, etc.) that are observed on top of the contribution by the mean $\mathrm{H} \mathrm{I}$ distribution.

We find the following:
1. There is a correlation between the $100 \mu \mathrm{m} I R A S$ flux and the percent polarization such that $P(\%) \leq(0.16 \pm$ $0.05) F_{100}$, which implies that $P(\%) \leq(0.13 \pm 0.03) N_{20}-$ 0.22 , where $N_{20}$ is the $\mathrm{H}$ I column density in units of $10^{20}$ $\mathrm{cm}^{-2}$. This relation is similar to that derived from the correlation between polarization and reddening (Spitzer 1978) apart from the constant term. This discrepancy is likely due to the uncertainties in the absolute calibration of the IRAS $100 \mu \mathrm{m}$ images.

2. The correlation between $I R A S$ and polarization data indicates that our survey is as deep as IRAS at least in the areas corresponding to low fluxes $\left(F_{100} \leq 8 \mathrm{MJy} \mathrm{sr}^{-1}\right)$ and to high fluxes $\left(F_{100} \geq 16 \mathrm{MJy} \mathrm{sr}^{-1}\right)$. The high flux limit corresponds to region $\mathrm{A}$ and the outskirts of the OrionMonoceros complex located at a distance of 500-700 pc. The low flux limit corresponds to high-latitude IRAS cirrus. Therefore, along these lines of sight, the magnetic field direction does not change significantly with the optical depth and our polarization survey is as deep as the IRAS. The intermediate region corresponds to the fields A3-A4 and B7-B10 that are located in regions where many smallscale features in the IRAS maps are detected.

3. The central finding of this paper is that the Galactic magnetic field at high latitudes is perpendicular to the stream in region B, and especially in regions where the dispersion in P.A. is smaller. The magnetic field is also perpendicular to the filamentary high-latitude cloud, MBM 16. We interpret this as evidence that the Galactic magnetic field toward the high-latitude clouds is predominantly toroidal in character, wrapping around the gas filaments.

4. There is a large component parallel to the stream in region A where the outskirts of the molecular cloud complex of Orion-Monoceros are sampled. The magnetic field is also parallel to the IRAS cirrus and the plume of dust that extends to high Galactic latitudes. This indicates that the field is predominantly poloidal nearer to the plane.

5. The dispersion in position angles of the polarization vectors is slightly higher than $0.4 \mathrm{rad}$ for both regions $\mathrm{A}$ and B. This may be due to the fact that we are dealing with a more diffuse medium than clouds located closer to the Galactic plane.

6. Our results can be interpreted in terms of an arcade of magnetic flux loops that rise out of the Galactic plane and up into the corona of the Galaxy. Such structures ought to acquire a force-free character dominated by toroidal magnetic field at high latitudes. Gas contained within these flux tubes drops back to the Galactic plane and this may explain the correlation of gas density, velocity, and magnetic structure that we have uncovered in our survey.

In order to further test some of our conclusions and their theoretical consequences, better measurements of the magnetic field structure toward the high-velocity clouds are needed. In particular, one would like, by spectroscopic observations, to have accurate distances to the background stars used in our survey.

Most of this work was carried out making use of the Computer Facilities at the ESA-IUE Observatory at VILSPA (Madrid, Spain). Ana I. Gómez de Castro thanks the VILSPA staff for their support while carrying out this project. We thank the support staffs of the Calar Alto (Spain) and Mont Mégantic Observatories (Canada) for their assistance in doing these observations. We thank an 
anonymous referee for useful remarks. This paper made use of the facilities provided by the Canadian Astronomical Data Center (CADC), and also those which support the SIMBAD database in France. A. I. G. de C. was supported in part by an NSERC International Postdoctoral Fellow- ship and a MEC "re-entry" fellowship. The research of R. E. P. and P. B. is supported by grants from the Natural Sciences and Engineering Research Council of Canada. The research of A. I. G. de C. is supported by the research grant PB 93-491.

\section{REFERENCES}

Angel, J. R. P., \& Landstreet, J. D. 1970, ApJ, 160, L147

Bajaja, E., Cappa de Nicolau, C. E., Cersosimo, J. C., Loiseau, N., Martin, M. C., Morras, R., Olano, C. A., \& Poppel, W. G. L. 1985, ApJS, 58, 143

Bally, J. 1989, in Low Mass Star Formation and Pre-Main-Sequence Objects, ed. B. Reipurth (Garching: ESO), 1

Bastien, P. 1991, in Physics of Star Formation and Early Stellar Evolution, ed. C. Lada \& N. Kylafis (Dordrecht: Kluwer), 709

Binney, J., \& Tremaine, S. 1987, Galactic Dynamics (Princeton: Princeton Univ. Press)

Blitz, L., Magnani, L., \& Wandel, A. 1988, ApJ, 331, L127

Blitz, L., \& Shu, F. H. 1980, ApJ, 238, 148

Boulanger, F. 1994, in The First Symposium on the Infrared Cirrus and Diffuse Interstellar Clouds, ed. R. M. Cutri \& William B. Latter (San Francisco: ASP), 101

Boulanger, F., Baud, B., \& van Albada, G. D. 1985, A\&A, 144, L9

Boulanger, F., \& Perault, M. 1988, ApJ, 330, 964 (BP)

Bregman, J. N. 1980, ApJ, 236, 577

Chandrasekhar, S., \& Kendall, P. C. 1957, ApJ, 126, 457

de Vries, C. P., \& Le Poole, R. S. 1985, A\&A, 145, L7

Dickey, J. M., \& Lockman, F. J. 1990, ARA\&A, 28, 215 (DL)

Elmegreen, B. G. 1982, ApJ, 253, 634

Foglizzo, T., \& Tagger, M. 1995, A\&A, 301, 293

Frisch, P. C. 1981, Nature, 293, 377

Giovanelli, R. 1980a, AJ, 85, 1155

.1980b, ApJ, 238, 554

Gómez de Castro, A. I., \& Pudritz, R. E. 1992, ApJ, 395, 501

Gómez de Castro, A. I., Pudritz, R. E., \& Bastien, P. 1991, in Evolution of Interstellar Matter and Dynamics of Galaxies, ed. J. Palous (Cambridge: Cambridge Univ. Press)

Heiles, C., Goodman, A. A., McKee, C. F., \& Zweibel, E. G. 1993, in Protostars and Planets III, ed. E. H. Levy \& J. I. Lunine (Tucson: Univ. Arizona Press), 279

Heyer, M. H., Vrba, F. J., Snell, R. L., Schloerb, F. P., Strom, S. E., \& Strom, K. M. 1987, ApJ, 321, 855

Heyvaerts, J., \& Priest, E. R. 1984, A\&A, 137, 63 (HP)

Hobbs, L. M., Blitz, L., Penprase, B. E., Magnani, L., \& Welty, D. E. 1988, ApJ, 327,356

Hulsbosh, A. N. M., \& Wakker, B. P. 1988, A\&AS, 75, 191

Klare, G., \& Neckel, T. 1977, A\&AS, 27, 215

Kulkarni, S. R., \& Heiles, C. 1988, in Galactic and Extragalactic Radio Astronomy, ed. G. L. Verschuur \& K. I. Kellermann (Berlin: Springer), A5

Levasseur-Regourd, A. C., Renard, J. B., \& Dumont, R. 1990, Icarus, 86, 264
Lockman, F. J., Hobbs, L. M., \& Shull, J. M. 1986, ApJ, 301, 380

Magnani, L., Blitz, L., \& Mundy, L. 1985, ApJ, 295, 402

Martin, P. G., Rogers, C., Reach, W. T., Dewdney, P. E., \& Heiles, C. E. 1994, in The First Symposium on the Infrared Cirrus and Diffuse Interstellar Clouds, ed. R. M. Cutri \& William B. Latter (San Francisco: ASP), 188

Mathewson, D. S., \& Ford, V. L. 1970, MmRAS, 74, 139

Matsumoto, R., Horiuchi, T., Hanawa, T., \& Shibata, K. 1988, PASJ, 40, 171

McCutcheon, W. H., Vrba, F. J., Dickman, R. L., \& Clemens, D. P. 1986, ApJ, 309, 619

McKee, C. F., \& Ostriker, J. P. 1977, ApJ, 218, 148

Mirabel, I. F. 1982, ApJ, 256, 112

Muller, C. A., Oort, J. H., \& Raimond, E. 1963, Comptes Rendues Acad. Sci. Paris, 257, 1661

Myers, P. C., \& Goodman, A. A. 1991, ApJ, 373, 509

Norman, C. A., \& Ikeuchi, S. 1989, ApJ, 345, 372

Odenwald, S. F. 1988, ApJ, 325, 320

Proetel, K. 1978, Thesis, Univ. Heidelberg

Raadu, M. A., \& Nakagawa, Y. 1971, Sol. Phys., 20, 64

Schwan, H. 1991, A\&A, 243, 386

Serkowski, K., Mathewson, D. S., \& Ford, V. L. 1975, ApJ, 196, 261

Snowden, S. L., Cox, D. P., McCammon, D., \& Sanders, W. T. 1990, ApJ, 354,211

Songaila, A., Cowie, L. L., \& Weaver, H. 1988, ApJ, 329, 580

Spitzer, L., Jr. 1978, Physical Processes in the Interstellar Medium (New York: Wiley)

Strom, S. E., Strom, K. M., \& Edwards, S. 1988 in Galactic and Extragalactic Star Formation, ed. R. Pudritz \& M. Fich (Dordrecht: Kluwer), 53

Tamanaha, C. M. 1996, ApJS, 104, 81

Tandberg-Hanssen, E., \& Emslie, A. G. 1988, The Physics of Solar Flares (New York: Cambridge Univ. Press)

Terebey, S., \& Fich, M. 1986, ApJ, 309, L73

Trapero, J., Beckman, J. E., Génova, R., \& McKeith, C. D. 1992, ApJ, 394, 552

Ungerechts, H., \& Thaddeus, P. 1987, ApJS, 63, 645

Wakker, B. P., \& Boulanger, F. 1986, A\&A, 170, 84

Wakker, B. P., \& Schwarz, U. J. 1991, A\&A, 250, 484

Wakker, B. P., \& van Woerden, H. 1991, A\&A, 250, 509

Whittet, D. C. B., Martin, P. G., Hough, J. H., Rouse, M. F., Bailey, J. A., \& Axon, D. J. 1992, ApJ, 386, 562 


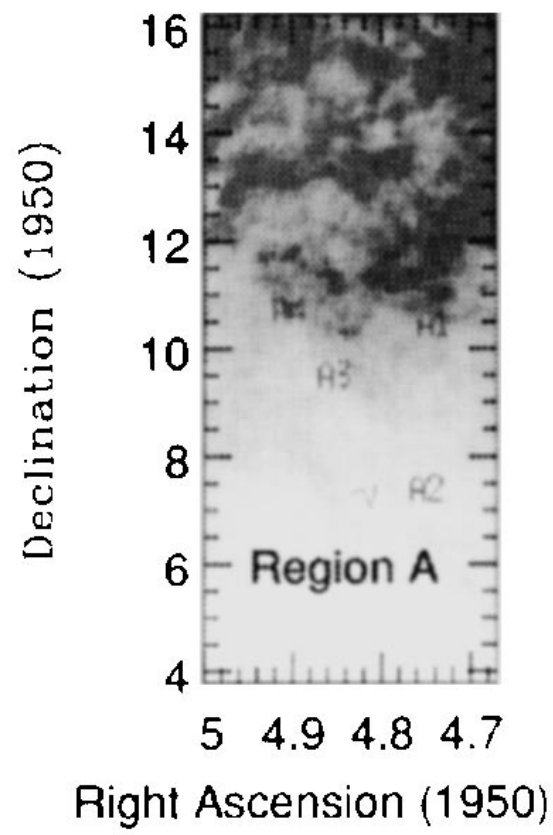

FIG. $1 a$

FIG. 1.-100 $\mu \mathrm{m}$ IRAS maps of the regions sampled in the polarization survey. (a) Region A and (b) region B. The location of the fields (see Table 1) is indicated. The overlapping of adjacent IRAS images is not good in the corners, this shows up in $b$ where some features located at high and low delta and close to the edges between two IRAS images are observed twice. The grayscale is set so white corresponds to a flux of $0 \mathrm{MJy} \mathrm{sr}^{-1}$ at $100 \mu \mathrm{m}$ and black corresponds to 20 and $15 \mathrm{MJy} \mathrm{sr}^{-1}$ for $a$ and $b$, respectively. North is up and east to the left.

GOMEZ DE CASTRO et al. (see 476, 718) 


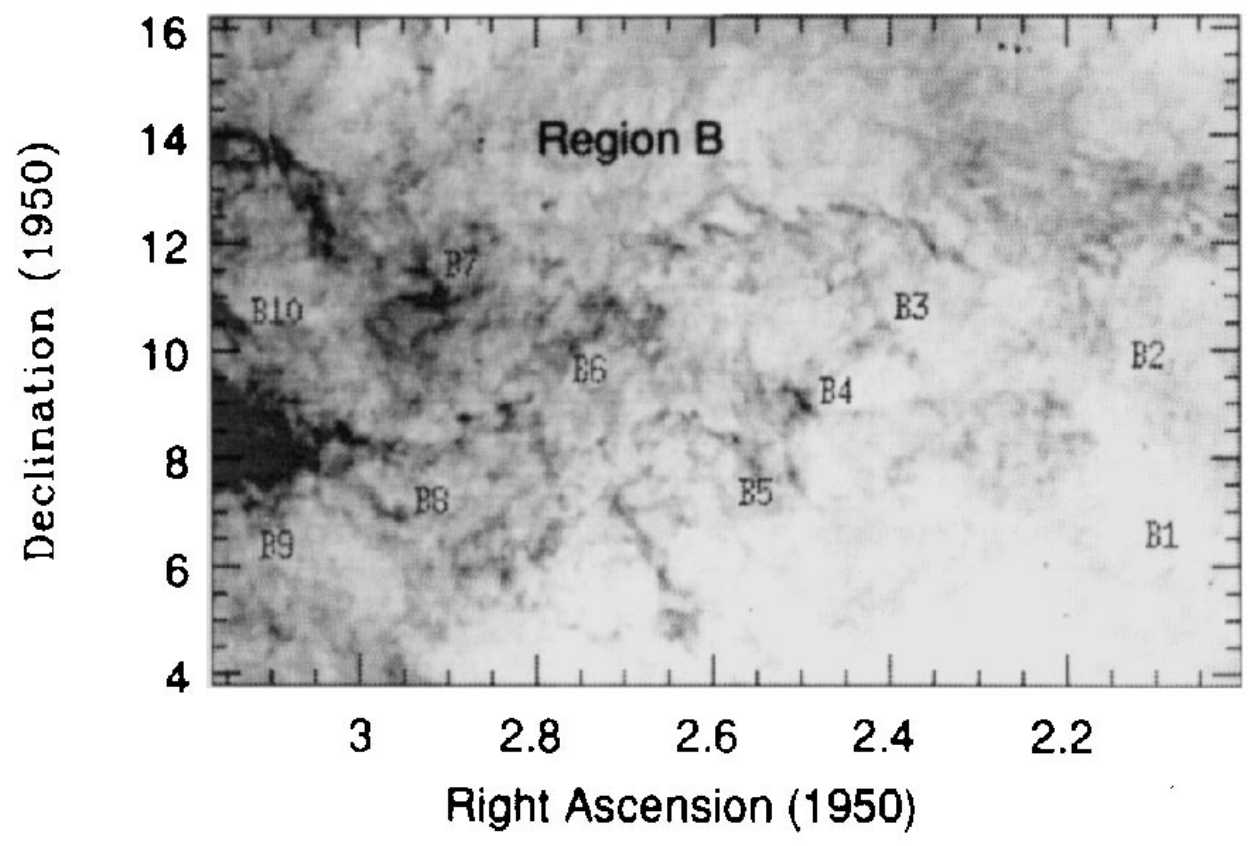

FIG. $1 b$

GOMEZ DE CASTRo et al. (see 476, 718) 